\title{
National Bureau of standards
}

Wibrary, N.W. BLdg

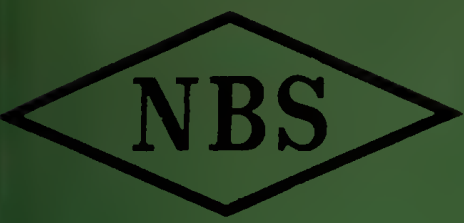

APR I 91963

\section{Eechnical Note}

\section{TABLES TO FACILITATE THE DETERMINATION} OF THE FERRIMAGNETIC RESONANCE LINEWIDTH OF NON-METALLIC MAGNETIC MATERIALS

CONSTANCE C. PRESTON AND WILLIAM E. CASE

U. S. DEPARTMENT OF COMMERCE NATIONAL BUREAU OF STANDARDS 


\section{THE NATIONAL BUREAU OF STANDARDS}

\section{Functions and Activities}

The functions of the National Bureau of Standards are set forth in the Act of Congress, March 3, 1901, as amended by Congress in Public Law 619, 1950. These include the development and maintenance of the national standards of measurement and the provision of means and methorls for making measurements consistent with these standards; the determination of physical constants and properties of materials; the development of methods and instrunents for testing materials, devices, and structures; advisory services to government agencies on scientific and technical problems; invention and development of devices to serve special needs of the Government; and the development of standard practices, codes, and specifications. 'The work includes basic and applied research, development, engineering, instrumentation, testing, evaluation, calibration services, and various consultation and information services. Research projects are also performed for other government agencies when the work relates to and supplements the basic program of the Bureau or when the Bureau's unique competence is required. The scope of activities is suggested by the listing of divisions and sections on the inside of the back cover.

\section{Publications}

The results of the Bureau's research are published either in the Bureau's own series of publications or in the journals of professional and scientific societies. The Bureau publishes three periodicals available from the Government Printing Office: The Journal of Research, published in four separate sections, presents complete scientific and teclunical papers; (he 'Tecllnical News Bulletin presents summary and preliminary reports on work in progress; and the Central Radio Propagation Laboratory Ionospleric Predictions provides data for detcrmining the best frequencies to use for radio communications throughout the world. There are also five series of nonperiodical publications: Monographs, Applied Mathematics Series, Handbools, Miscellaneous Publications, and Technical Notes.

A complete listing of the Bureau's publications can be found in National Bureau of Standards Circular 460, Publications of the National Bureau of Standards, 1901 to June 1947 (\$1.25), and the Supplement to National Bureau of Standards Circular 460, July 1947 to June 1957 (\$1.50), and Miscellaneous Publication 240, July 1957 to June 1960 (includes 'Titles of Paper's Published in Outside Journals 1950 to 1959) (\$2.25); available from the Superintendent of Documents, Government Printing Office, Washington 25, D.C. 


\title{
NATIONAL BUREAU OF STANDARDS \\ Eechnical Note 173 \\ ISSUED APRIL 15, 1963
}

\section{TABLES TO FACILITATE THE DETERMINATION \\ OF THE FERRIMAGNETIC RESONANCE LINEWIDTH \\ OF NON-METALLIC MAGNETIC MATERIALS}

\author{
Constance C. Preston and William E. Case \\ Radio Standards Laboratory \\ National Bureau of Standards \\ Boulder, Colorado
}

NBS Technical Notes are designed to supplement the Bureau's regular publications program. They provide a means for making available scientific data that are of transient or limited interest. Technical Notes may be listed or referred to in the open literature. 


\section{Contents}

Page

Introduction - . . . . . . . . . . . . 1

Method _ . . . . . . . . . . . . . 2

References _. - $\ldots \ldots$ - 6

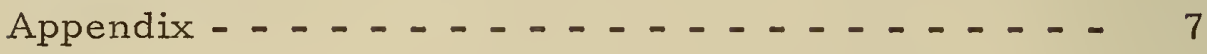


Tables to Facilitate the Determination of the Ferrimagnetic

Resonance Linewidth of Non-Metallic Magnetic Materials

\section{Constance C. Preston and William E. Case}

A common test procedure for measuring ferrimagnetic resonance linewidth, $\Delta H$, and gyromagnetic ratio, $\gamma$, of microwave ferrites is based upon a perturbation analysis of the complex frequency shift obtained when a small sample is placed in a resonant cavity and an applied dc magnetic field. However, this method necessitates the plotting of a ferrimagnetic resonance loss curve. To find $\Delta \mathrm{H}$ it is possible to derive an equation using lumped circuit theory which reduces the number of needed measurements to four attenuator readings.

This paper provides a table which gives values of attenuation according to the following formula which facilitates the determination of linewidth:

$$
A=20 \log 2-20 \log \left(10^{\frac{A_{o}-A_{r}}{20}}+1\right) \text { for } A_{o}-A_{r}=0.41(0.01) 32.40 .
$$

\section{INTRODUCTION}

Microwave devices such as isolators, phase shifters, and circulators utilize ferrite materials which exhibit ferrimagnetic resonance phenomena at microwave frequencies. Two important properties of ferrites at these frequencies are the effective gyromagnetic ratio, $\gamma$, and the ferrimagnetic resonance linewidth, $\Delta \mathrm{H}$.

Both of these properties of a ferrite material can be determined by inserting a small sample in a cavity and measuring the trans mission loss of the system. It is assumed that the sample is sufficiently small for the perturbation theory to be applicable. Measurements may be made by holding the input power to the cavity constant, varying the applied dc field, measuring the complex resonant frequency with and without the sample, and plotting the imaginary susceptibility curve. Linewidth is defined as the separation of the two dc field values at which the power absorbed by the ferrite sample is one half the maximum absorption.

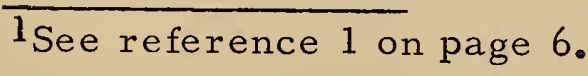


The above method requires a number of measurements to complete the curve. By using the accepted expressions for the relationship between the $Q$ and transmission loss of a loaded and unloaded resonant cavity ${ }^{2}$, it is possible to derive a formula (see Appendix I) whereby the linewidth can be determined by making only four measurements. The Non-Metallic Magnetic Materials Subcommittee VII of ASTM, Committee C21, has proposed specifications for this method in which spherical-shaped ferrite samples are measured in a rectangular $\mathrm{TE}_{102}$ resonant cavity.

\section{METHOD}

A block diagram of the experimental setup used for this method is shown in Figure 1. A constant input power to the precision calibrated attenuator as monitored by the power detector is assumed. The frequency is adjusted to give cavity resonance for the empty cavity, and the precision attenuator is set to $A_{0}$ to give a convenient output reference level as indicated on the output detector. The sample is then inserted, the dc magnetic field adjusted for maximum absorption in the sample, the frequency adjusted for cavity resonance, and the precision attenuator set to obtain the same output reference signal. The new attenuator $r$ eading is defined as $A_{r}$, and the dc field reading is defined as $\mathrm{H}_{\mathrm{r}}$.

The attenuator reading corresponding to the half-power points on the ferrimagnetic resonance loss curve of the sample is calculated from the following formula as derived on page 12 of the Appendix:

$$
A_{\frac{1}{2}}=A_{0}+20 \log 2-20 \log \left(\frac{A_{0}-A_{r}}{20}+1\right) .
$$

The attenuator is now set at the calculated $\mathrm{A}_{\frac{1}{2}}$ value. The dc magnetic field necessary to return the output power to its previous level on one side of resonance is then read and recorded. The dc field is then similarly adjusted and recorded for the other side of resonance. The difference between these two dc field measurements is the linewidth, $\Delta \mathrm{H}$. For all measurements, the frequency must be adjusted to cavity resonance.

Calculating $A_{\frac{1}{2}}$ each time a measurement is made is timeconsuming and prone to error. Time delay between maximum loss and half-power measurements can introduce error due to equipment drift. Faster and more accurate data can be taken using the following

$\overline{2_{\text {See }} \text { reference } 2}$ on page 6. 
table which gives values of $\mathrm{A}$ for $\mathrm{A}_{\mathrm{O}}-\mathrm{A}_{\mathrm{r}}$ from .41 to 32.40 decibels where $A$ is defined as:

$$
A=20 \log 2-20 \log \left(10^{\frac{A_{0}-A_{r}}{20}}+1\right) \text {, }
$$

and thus,

$$
\mathrm{A}_{\frac{1}{2}}=\mathrm{A}_{0}+\mathrm{A}
$$

The above technique is essentially an rf substitution method. However, an IF substitution method as shown in Figure 2 may be used for greater accuracy if a $30 \mathrm{Mc} / \mathrm{s}$ high precision piston attenuator is available because, generally, a lower frequency IF piston attenuator can be made more accurate than an rf attenuator. The IF system has the advantage of greater accuracy, greater sensitivity, and that the same IF attenuator can be used for different rf frequencies. Disadvantages of the system are that more equipment, more complicated tuning, and separate local oscillators are required for each frequency. The piston attenuator is inserted between the cavity and the output detector. The procedure is the same as for the rf substitution method except that the input power to the cavity is held constant, and the power level to the IF detector is maintained constant by adjusting the piston attenuator.

The effective gyromagnetic ratio, $\gamma$, is determined from the sphere measurement data by the formula,

$$
\gamma=\frac{2 \pi f}{H_{r}},
$$

where $f_{r}$ is the microwave frequency required for cavity resonance at maximum sample absorption, and $\mathrm{H}_{\mathrm{r}}$ is the corresponding dc field. The gyromagnetic ratio is sometimes expressed in terms of the apparent $g$ factor in which case the above expression becomes

$$
g=\frac{f_{r}}{1.3995 \mathrm{H}_{r}} \cong \frac{f_{r}}{1.4 \mathrm{H}_{r}} \text {, }
$$

if $\mathrm{f}_{\mathrm{r}}$ is in $\mathrm{Mc}$ and $\mathrm{H}_{\mathrm{r}}$ is in oersteds. 


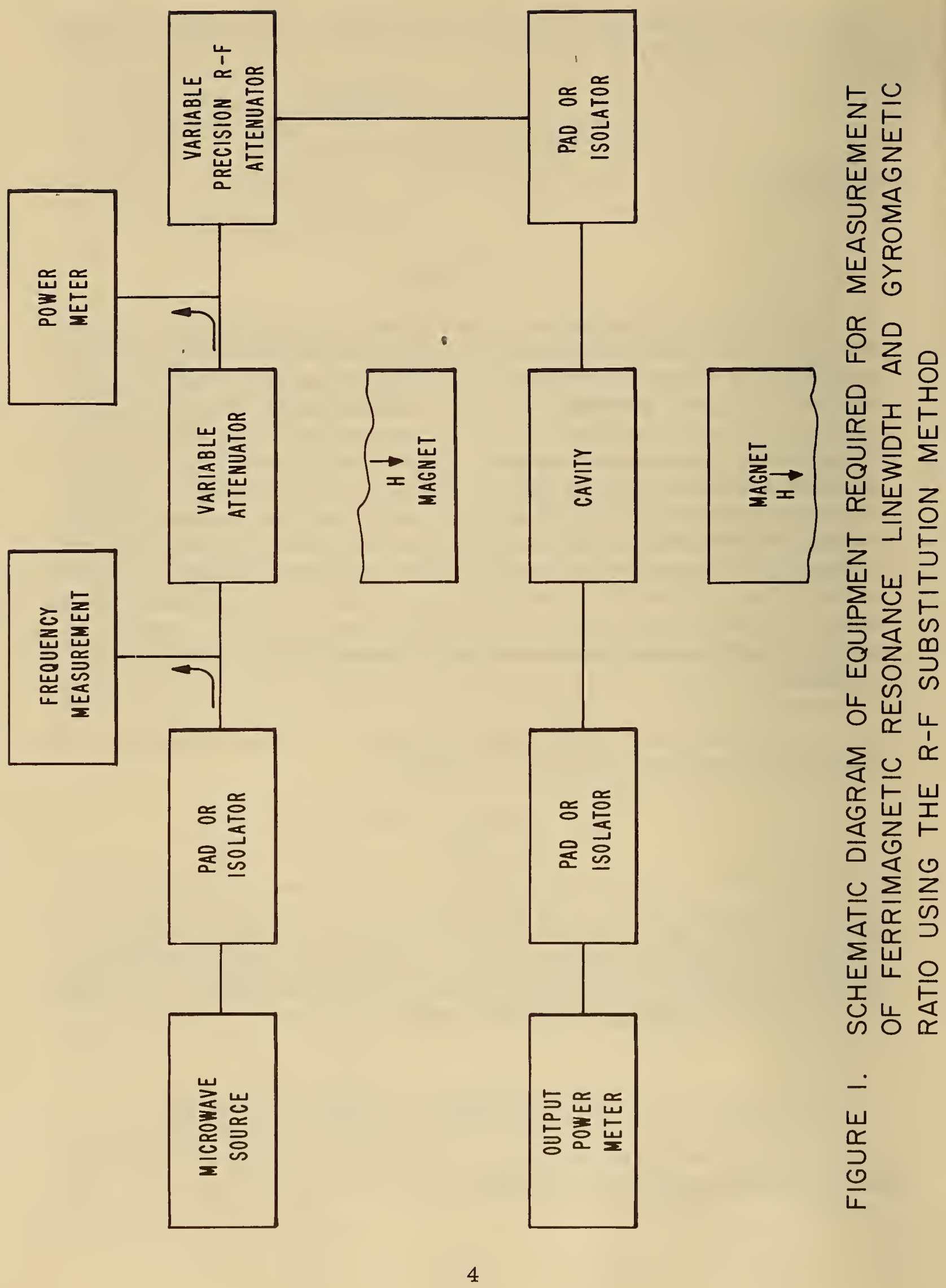




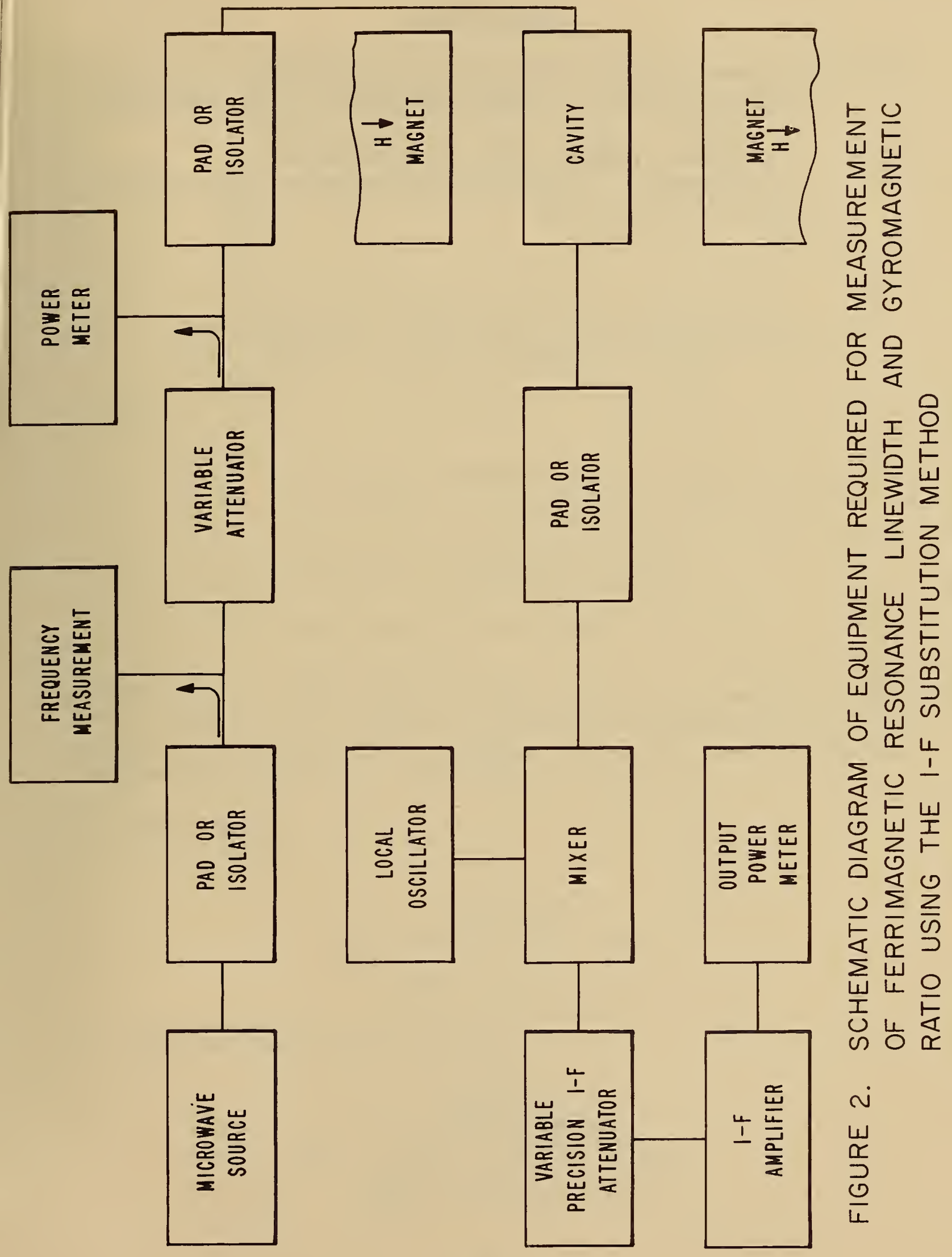




\section{REFERENCES}

1. R. F. Soohoo, "Theory and Application of Ferrites," p. 260-263, (Prentice-Hall, Inc., Englewood Cliffs, New Jersey, 1960).

2. C. G. Montgomery (ed.), "Techniques of Microwave Measurements," p. 289-291, (McGraw-Hill Book Company, Inc., New York, 1947). 


\section{APPENDIX}

The equivalent circuit for a transmission microwave cavity is commonly shown as in Figure 3 (see reference 2).

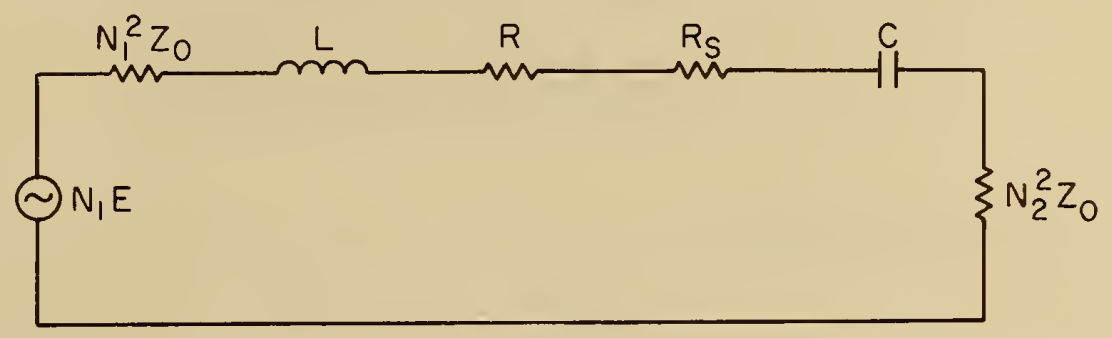

FIG. 3 EQUIVALENT CIRCUIT FOR TRANSMISSION LINE CAVITY COUPLED TO MATCHED GENERATOR AND LOAD

where

$$
\begin{aligned}
& \mathrm{R} \quad=\text { resistance representing loss of empty cavity } \\
& \mathrm{R}_{\mathrm{s}} \quad=\text { resistance representing loss due to sample } \\
& \mathrm{N}_{1}^{2} \mathrm{Z}_{\mathrm{o}}=\text { resistance of matched generator } \\
& \mathrm{N}_{2}^{2} \mathrm{Z}_{\mathrm{o}}=\text { resistance of matched load }
\end{aligned}
$$

Assume that the output power is kept constant by varying the input power (input voltage $\mathrm{N}_{1} \mathrm{E}$ ) by means of a matched precision adjustable attenuator. We now write the power $\left[\mathrm{P}_{\text {load }}=\mathrm{N}_{2}{ }^{2} \mathrm{Z}_{\mathrm{O}}|\mathrm{I}|^{2}\right]$ delivered to the load for the following three conditions:

(1) Empty cavity $E_{1}=E_{1} ; R_{s}=0$

$$
P_{a}=\frac{N_{1}{ }^{2} N_{2}{ }^{2} E_{1}{ }^{2} Z_{0}}{\left[\left(N_{1}{ }^{2}+N_{2}{ }^{2}\right) Z_{0}+R\right]}
$$

(2) Sample at maximum loss $E=E_{2}$

$$
P_{b}=\frac{N_{1}{ }^{2} N_{2}{ }^{2} E_{2}{ }^{2} Z_{0}}{\left[\left(N_{1}{ }^{2}+N_{2}{ }^{2}\right) Z_{o}+R+R_{s}\right]}
$$


(3) Sample at $\frac{1}{2}$ maximum loss $\mathrm{E}=\mathrm{E}_{3}$

$$
P_{c}=\frac{N_{1}{ }^{2} N_{2}{ }^{2} E_{3}{ }^{2} Z_{0}}{\left[\left(N_{1}{ }^{2}+N_{2}{ }^{2}\right) Z_{0}+R+\frac{R_{s}}{2}\right]} .
$$

Since $P_{a}=P_{b}$ and $P_{b}=P_{c}$,

$$
\begin{aligned}
& \frac{E_{1}}{E_{2}}=\frac{\left(N_{1}{ }^{2}+N_{2}{ }^{2}\right) Z_{0}+R}{\left(N_{1}{ }^{2}+N_{2}{ }^{2}\right) Z_{0}+R+R}=K_{1}=\frac{\sqrt{P_{1}}}{\sqrt{P_{2}}} \\
& \frac{E_{2}}{E_{3}}=\frac{\left(N_{1}^{2}+N_{2}^{2}\right) Z_{0}+R+R s}{\left(N_{1}{ }^{2}+N_{2}{ }^{2}\right) Z_{0}+R+\frac{R_{s}}{2}}=K_{2}=\frac{\sqrt{P_{2}}}{\sqrt{P_{3}}},
\end{aligned}
$$

where $P_{1}, P_{2}$ and $P_{3}$ are the powers delivered by the attenuator for the three conditions.

Let

$$
\begin{gathered}
\left(\mathrm{N}_{1}{ }^{2}+\mathrm{N}_{2}{ }^{2}\right) \mathrm{Z}_{0}+\mathrm{R}=\mathrm{B} \\
\frac{\mathrm{B}}{\mathrm{B}+\mathrm{R}_{\mathrm{s}}}=\mathrm{K}_{1} \\
\mathrm{~B}=\mathrm{K}_{1} \mathrm{~B}+\mathrm{K}_{1} \mathrm{R}_{\mathrm{s}} \\
\mathrm{B}\left(1-\mathrm{K}_{1}\right)=\mathrm{K}_{1} \mathrm{R}_{\mathrm{s}} \\
\frac{\mathrm{B}+\mathrm{R}_{\mathrm{s}}}{\mathrm{B}+\frac{\mathrm{R}}{2}}=\mathrm{K}_{2}
\end{gathered}
$$




$$
\begin{aligned}
& B=K_{2} B+\frac{K_{2} R_{s}}{2}-R_{s} \\
& B\left(1-K_{2}\right)=\frac{R_{s}}{2}\left(K_{2}-2\right) \\
& \frac{\mathrm{R}_{s}\left(\mathrm{~K}_{2}-2\right)}{2\left(1-\mathrm{K}_{2}\right)}=\frac{\mathrm{K}_{1} \mathrm{R}_{s}}{1-\mathrm{K}_{1}} \\
& \mathrm{~K}_{2}-2-\mathrm{K}_{1} \mathrm{~K}_{2}+2 \mathrm{~K}_{1}=2 \mathrm{~K}_{1}-2 \mathrm{~K}_{1} \mathrm{~K}_{2} \\
& \mathrm{~K}_{2}+\mathrm{K}_{1} \mathrm{~K}_{2}=2 \\
& \mathrm{~K}_{2}=\frac{2}{1+\mathrm{K}_{1}} \\
& \frac{\sqrt{\mathrm{P}_{2}}}{\sqrt{\mathrm{P}_{3}}}=\frac{2}{1+\frac{\sqrt{\mathrm{P}_{1}}}{\sqrt{\mathrm{P}_{2}}}} \\
& \frac{\mathrm{P}_{2}}{\mathrm{P}_{3}}=\left(\frac{2}{1+\frac{\sqrt{\mathrm{P}_{1}}}{\sqrt{\mathrm{P}_{2}}}}\right)^{2} .
\end{aligned}
$$

Referring to Figure 4, we may develop the relationships between the various power levels and the corresponding attenuator readings. 


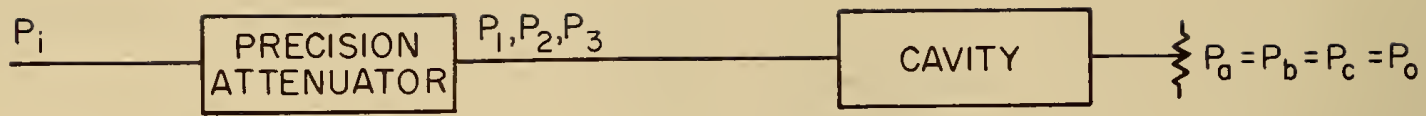

FIG. 4 BLOCK DIAGRAM INDICATING POWER RELATIONSHIPS

where

$$
\begin{aligned}
& P_{i}=\text { input power delivered to precision attenuator. } \\
& P_{0}=\text { output power delivered to load. }
\end{aligned}
$$

Assume $P_{i}$ and $P_{0}$ are held constant.

For empty cavity, let

$$
A_{0}=10 \log \frac{P_{i}}{P_{1}}=10 \log P_{i}-10 \log P_{1} \text {. }
$$

For cavity with sample at maximum loss, let

$$
A_{r}=10 \log \frac{P_{i}}{P_{2}}=10 \log P_{i}-10 \log P_{2}
$$

For cavity with sample at $\frac{1}{2}$ maximum loss, let

$$
\begin{array}{r}
A_{\frac{1}{2}}=10 \log \frac{P_{i}}{P_{3}}=10 \log P_{i}-10 \log P_{3} \\
A_{0}-A_{r}=10 \log P_{2}-10 \log P_{1}=10 \log \frac{P_{2}}{P_{1}} \\
A_{\frac{1}{2}}-A_{r}=10 \log P_{2}-10 \log P_{3}=10 \log \frac{P_{2}}{P_{3}} .
\end{array}
$$

From above

$$
10 \log \frac{P_{2}}{P_{1}}=A_{0}-A_{r}
$$




$$
\begin{aligned}
& 20 \log \frac{\sqrt{P_{2}}}{\sqrt{P_{1}}}=A_{O}-A_{r} \\
& \log \frac{\sqrt{\mathrm{P}_{2}}}{\sqrt{\mathrm{P}_{1}}}=\frac{\mathrm{A}_{0}-\mathrm{A}_{\mathrm{r}}}{20} \\
& \frac{\sqrt{P_{2}}}{\sqrt{P_{1}}}=10^{\frac{A_{0}-A_{r}}{20}} \\
& A_{\frac{1}{2}}-A_{0}=A_{\frac{1}{2}}-A_{r}-\left(A_{0}-A_{r}\right) \\
& A_{\frac{1}{2}}-A_{0}=10 \log \frac{P_{2}}{P_{3}}-10 \log \frac{P_{2}}{P_{1}} \\
& A_{\frac{1}{2}}-A_{0}=20 \log \frac{2}{1+\frac{\sqrt{P_{1}}}{\sqrt{\mathrm{P}_{2}}}}-20 \log \frac{\sqrt{\mathrm{P}_{2}}}{\sqrt{\mathrm{P}_{1}}} \\
& A_{\frac{1}{2}}-A_{0}=20 \log 2-20 \log \left(\frac{\sqrt{P_{2}}}{\sqrt{P_{1}}}+1\right) \\
& A_{\frac{1}{2}}=A_{0}+20 \log 2-20 \log \left[10^{\frac{A_{0}-A_{r}}{20}}+1\right] \text {, }
\end{aligned}
$$

which is the working equation given in the text of this paper. 


\section{ATTENUATOR READINGS}

A - empty cavity

$A_{r}$ - sample in cavity--maximum absorption of sample

$\mathrm{A}_{\frac{1}{2}}$ - sample in cavity-- one half maximum absorption of sample

\section{WORKING EQUATIONS}

$$
A_{\frac{1}{2}}=A_{0}+20 \log 2-20 \log \left(10^{\frac{A_{0}-A_{r}}{20}}+1\right)
$$

or

$$
A_{\frac{1}{2}}=A_{0}+A
$$

Tables give values of $\mathrm{A}$ as a function of $\mathrm{A}_{\mathrm{o}}-\mathrm{A}_{\mathrm{r}}$. 


\begin{tabular}{|c|c|c|c|c|c|c|c|}
\hline$A_{0}{ }^{-A_{r}}$ & A & $A_{0}-A_{r}$ & A & $A_{0}-A_{r}$ & A & $A_{0}-A_{r}$ & A \\
\hline 0.41 & -0.21 & 0.81 & -0.41 & 1.21 & -0.63 & 1.61 & -0.84 \\
\hline 0.42 & -0.21 & 0.82 & -0.42 & 1.22 & -0.63 & 1.62 & -0.85 \\
\hline 0.43 & -0.22 & 0.83 & -0.43 & 1.23 & -0.64 & 1.63 & -0.85 \\
\hline 0.44 & -0.22 & 0.84 & -0.43 & 1.24 & -0.64 & 1.64 & -0.86 \\
\hline 0.45 & -0.23 & 0.85 & -0.44 & 1.25 & -0.65 & 1.65 & -0.86 \\
\hline 0.46 & -0.23 & 0.86 & -0.44 & 1.26 & -0.65 & 1.66 & -0.87 \\
\hline 0.47 & -0.24 & 0.87 & -0.45 & 1.27 & -0.66 & 1.67 & -0.88 \\
\hline 0.48 & -0.24 & 0.88 & -0.45 & 1.28 & -0.66 & 1.68 & -0.88 \\
\hline 0.49 & -0.25 & 0.89 & -0.46 & 1.29 & -0.67 & 1.69 & -0.89 \\
\hline 0.50 & -0.25 & 0.90 & -0.46 & 1.30 & -0.67 & 1.70 & -0.89 \\
\hline 0.51 & -0.26 & 0.91 & -0.47 & 1.31 & -0.68 & 1.71 & -0.90 \\
\hline 0.52 & -0.26 & 0.92 & -0.47 & 1.32 & -0.69 & 1.72 & -0.90 \\
\hline 0.53 & -0.27 & 0.93 & -0.48 & 1.33 & -0.69 & 1.73 & -0.91 \\
\hline 0.54 & -0.27 & 0.94 & -0.48 & 1.34 & -0.70 & 1.74 & -0.91 \\
\hline 0.55 & -0.28 & 0.95 & -0.49 & 1.35 & -0.70 & 1.75 & -0.92 \\
\hline 0.56 & -0.29 & 0.96 & -0.49 & 1.36 & -0.71 & 1.76 & -0.93 \\
\hline 0.57 & -0.29 & 0.97 & -0.50 & 1.37 & -0.71 & 1.77 & -0.93 \\
\hline 0.58 & -0.30 & 0.98 & -0.50 & 1.38 & -0.72 & 1.78 & -0.94 \\
\hline 0.59 & -0.30 & 0.99 & -0.51 & 1.39 & -0.72 & 1.79 & -0.94 \\
\hline 0.60 & -0.31 & 1.00 & -0.51 & 1.40 & -0.73 & 1.80 & -0.95 \\
\hline 0.61 & -0.31 & 1.01 & -0.52 & 1.41 & -0.73 & 1.81 & -0.95 \\
\hline 0.62 & -0.32 & 1.02 & -0.53 & 1.42 & -0.74 & 1.82 & -0.96 \\
\hline 0.63 & -0.32 & 1.03 & -0.53 & 1.43 & -0.74 & 1.83 & -0.96 \\
\hline 0.64 & -0.33 & 1.04 & -0.54 & 1.44 & -0.75 & 1.84 & -0.97 \\
\hline 0.65 & -0.33 & 1.05 & -0.54 & 1.45 & -0.76 & 1.85 & -0.97 \\
\hline 0.66 & -0.34 & 1.06 & -0.55 & 1.46 & -0.76 & 1.86 & -0.98 \\
\hline 0.67 & -0.34 & 1.07 & -0.55 & 1.47 & -0.77 & 1.87 & -0.99 \\
\hline 0.68 & -0.35 & 1.08 & -0.56 & 1.48 & -0.77 & 1.88 & -0.99 \\
\hline 0.69 & -0.35 & 1.09 & -0.56 & 1.49 & -0.78 & 1.89 & -1.00 \\
\hline 0.70 & -0.36 & 1.10 & -0.57 & 1.50 & -0.78 & 1.90 & -1.00 \\
\hline 0.71 & -0.36 & 1.11 & -0.57 & 1.51 & -0.79 & 1.91 & -1.01 \\
\hline 0.72 & -0.37 & 1.12 & -0.58 & 1.52 & -0.79 & 1.92 & -1.01 \\
\hline 0.73 & -0.37 & 1.13 & -0.58 & 1.53 & -0.80 & 1.93 & -1.02 \\
\hline 0.74 & -0.38 & 1.14 & -0.59 & 1.54 & -0.80 & 1.94 & -1.02 \\
\hline 0.75 & -0.38 & 1.15 & -0.59 & 1.55 & -0.81 & 1.95 & -1.03 \\
\hline 0.76 & -0.39 & 1.16 & -0.60 & 1.56 & -0.82 & 1.96 & -1.04 \\
\hline 0.77 & -0.39 & 1.17 & -0.61 & 1.57 & -0.82 & 1.97 & -1.04 \\
\hline 0.78 & -0.40 & 1.18 & -0.61 & 1.58 & -0.83 & 1.98 & -1.05 \\
\hline 0.79 & -0.40 & 1.19 & -0.62 & 1.59 & -0.83 & 1.99 & -1.05 \\
\hline 0.80 & -0.41 & 1.20 & -0.62 & 1.60 & -0.84 & 2.00 & -1.06 \\
\hline
\end{tabular}




$\begin{array}{llllllll}2.01 & -1.06 & 2.41 & -1.29 & 2.81 & -1.52 & 3.21 & -1.75 \\ 2.02 & -1.07 & 2.42 & -1.29 & 2.82 & -1.52 & 3.22 & -1.76 \\ 2.03 & -1.07 & 2.43 & -1.30 & 2.83 & -1.53 & 3.23 & -1.76 \\ 2.04 & -1.08 & 2.44 & -1.31 & 2.84 & -1.54 & 3.24 & -1.77 \\ 2.05 & -1.09 & 2.45 & -1.31 & 2.85 & -1.54 & 3.25 & -1.78 \\ 2.06 & -1.09 & 2.46 & -1.32 & 2.86 & -1.55 & 3.26 & -1.78 \\ 2.07 & -1.10 & 2.47 & -1.32 & 2.87 & -1.55 & 3.27 & -1.79 \\ 2.08 & -1.10 & 2.48 & -1.33 & 2.88 & -1.56 & 3.28 & -1.79 \\ 2.09 & -1.11 & 2.49 & -1.33 & 2.89 & -1.57 & 3.29 & -1.80 \\ 2.10 & -1.11 & 2.50 & -1.34 & 2.90 & -1.57 & 3.30 & -1.81 \\ 2.11 & -1.12 & 2.51 & -1.35 & 2.91 & -1.58 & 3.31 & -1.81 \\ 2.12 & -1.13 & 2.52 & -1.35 & 2.92 & -1.58 & 3.32 & -1.82 \\ 2.13 & -1.13 & 2.53 & -1.36 & 2.93 & -1.59 & 3.33 & -1.82 \\ 2.14 & -1.14 & 2.54 & -1.36 & 2.94 & -1.59 & 3.34 & -1.83 \\ 2.15 & -1.14 & 2.55 & -1.37 & 2.95 & -1.60 & 3.35 & -1.84 \\ 2.16 & -1.15 & 2.56 & -1.37 & 2.96 & -1.61 & 3.36 & -1.84 \\ 2.17 & -1.15 & 2.57 & -1.38 & 2.97 & -1.61 & 3.37 & -1.85 \\ 2.18 & -1.16 & 2.58 & -1.39 & 2.98 & -1.62 & 3.38 & -1.85 \\ 2.19 & -1.16 & 2.59 & -1.39 & 2.99 & -1.62 & 3.39 & -1.86 \\ 2.20 & -1.17 & 2.60 & -1.40 & 3.00 & -1.63 & 3.40 & -1.87 \\ 2.21 & -1.18 & 2.61 & -1.40 & 3.01 & -1.64 & 3.41 & -1.87 \\ 2.22 & -1.18 & 2.62 & -1.41 & 3.02 & -1.64 & 3.42 & -1.88 \\ 2.23 & -1.19 & 2.63 & -1.41 & 3.03 & -1.65 & 3.43 & -1.88 \\ 2.24 & -1.19 & 2.64 & -1.42 & 3.04 & -1.65 & 3.44 & -1.89 \\ 2.25 & -1.20 & 2.65 & -1.43 & 3.05 & -1.66 & 3.45 & -1.90 \\ 2.26 & -1.20 & 2.66 & -1.43 & 3.06 & -1.66 & 3.46 & -1.90 \\ 2.27 & -1.21 & 2.67 & -1.44 & 3.07 & -1.67 & 3.47 & -1.91 \\ 2.28 & -1.22 & 2.68 & -1.44 & 3.08 & -1.68 & 3.48 & -1.91 \\ 2.29 & -1.22 & 2.69 & -1.45 & 3.09 & -1.68 & 3.49 & -1.92 \\ 2.30 & -1.23 & 2.70 & -1.46 & 3.10 & -1.69 & 3.50 & -1.93 \\ 2.31 & -1.23 & 2.71 & -1.46 & 3.11 & -1.69 & 3.51 & -1.93 \\ 2.32 & -1.24 & 2.72 & -1.47 & 3.12 & -1.70 & 3.52 & -1.94 \\ 2.33 & -1.24 & 2.73 & -1.47 & 3.13 & -1.71 & 3.53 & -1.94 \\ 2.34 & -1.25 & 2.74 & -1.48 & 3.14 & -1.71 & 3.54 & -1.95 \\ 2.35 & -1.25 & 2.75 & -1.48 & 3.15 & -1.72 & 3.55 & -1.96 \\ 2.36 & -1.26 & 2.76 & -1.49 & 3.16 & -1.72 & 3.56 & -1.96 \\ 2.37 & -1.27 & 2.77 & -1.50 & 3.17 & -1.73 & 3.57 & -1.97 \\ 2.38 & -1.27 & 2.78 & -1.50 & 3.18 & -1.74 & 3.58 & -1.97 \\ 2.39 & -1.28 & 2.79 & -1.51 & 3.19 & -1.74 & 3.59 & -1.98 \\ 2.40 & -1.28 & 2.80 & -1.51 & 3.20 & -1.75 & 3.60 & -1.99\end{array}$



$A_{0}-A_{r}$
A
$A_{0}{ }^{-A_{r}}$
A
$A_{0}-A_{r}$
A
$A_{0}-A_{r}$
A

\begin{tabular}{|c|c|c|c|c|c|c|c|}
\hline 3.61 & -1.99 & 4.01 & $-2 \cdot 23$ & 4.41 & $-2 \cdot 48$ & 4.81 & -2.73 \\
\hline 3.62 & -2.00 & 4.02 & -2.24 & 4.42 & -2.49 & 4.82 & -2.74 \\
\hline 3.63 & -2.00 & 4.03 & -2.25 & 4.43 & -2.49 & 4.83 & -2.75 \\
\hline 3.64 & -2.01 & 4.04 & -2.25 & 4.44 & -2.50 & 4.84 & -2.75 \\
\hline 3.65 & -2.02 & 4.05 & $-2 \cdot 26$ & 4.45 & -2.51 & 4.85 & -2.76 \\
\hline 3.66 & -2.02 & 4.06 & -2.27 & 4.46 & -2.51 & 4.86 & -2.77 \\
\hline 3.67 & -2.03 & 4.07 & -2.27 & $4 \cdot 47$ & -2.52 & 4.87 & -2.77 \\
\hline 3.68 & -2.03 & 4.08 & $-2 \cdot 28$ & 4.48 & -2.53 & 4.88 & -2.78 \\
\hline 3.69 & -2.04 & 4.09 & $-2 \cdot 28$ & 4.49 & -2.53 & 4.89 & -2.79 \\
\hline 3.70 & -2.05 & 4.10 & -2.29 & 4.50 & -2.54 & 4.90 & -2.79 \\
\hline 3.71 & -2.05 & 4.11 & $-2 \cdot 30$ & 4.51 & -2.54 & 4.91 & -2.80 \\
\hline 3.72 & -2.06 & 4.12 & $-2 \cdot 30$ & 4.52 & -2.55 & 4.92 & -2.80 \\
\hline 3.73 & -2.06 & 4.13 & $-2 \cdot 31$ & 4.53 & -2.56 & 4.93 & -2.81 \\
\hline 3.74 & -2.07 & 4.14 & $-2 \cdot 31$ & 4.54 & -2.56 & 4.94 & -2.82 \\
\hline 3.75 & -2.08 & 4.15 & $-2 \cdot 32$ & 4.55 & -2.57 & 4.95 & -2.82 \\
\hline 3.76 & -2.08 & 4.16 & $-2 \cdot 33$ & 4.56 & $-2 \cdot 58$ & 4.96 & -2.83 \\
\hline 3.77 & -2.09 & 4.17 & -2.33 & 4.57 & -2.58 & 4.97 & -2.84 \\
\hline 3.78 & -2.09 & 4.18 & -2.34 & 4.58 & -2.59 & 4.98 & -2.84 \\
\hline 3.79 & $-2 \cdot 10$ & 4.19 & -2.35 & 4.59 & -2.60 & 4.99 & -2.85 \\
\hline 3.80 & -2.11 & 4.20 & -2.35 & 4.60 & -2.60 & 5.00 & -2.86 \\
\hline 3.81 & -2.11 & 4.21 & $-2 \cdot 36$ & 4.61 & $-2 \cdot 61$ & 5.01 & -2.86 \\
\hline 3.82 & -2.12 & 4.22 & $-2 \cdot 36$ & 4.62 & -2.61 & 5.02 & -2.87 \\
\hline 3.83 & -2.12 & 4.23 & -2.37 & 4.63 & -2.62 & 5.03 & -2.87 \\
\hline 3.84 & -2.13 & 4.24 & $-2 \cdot 38$ & 4.64 & -2.63 & 5.04 & -2.88 \\
\hline 3.85 & $-2 \cdot 14$ & 4.25 & $-2 \cdot 38$ & 4.65 & -2.63 & 5.05 & -2.89 \\
\hline 3.86 & -2.14 & 4.26 & -2.39 & 4.66 & -2.64 & 5.06 & -2.89 \\
\hline 3.87 & $-2 \cdot 15$ & 4.27 & -2.40 & 4.67 & -2.65 & 5.07 & -2.90 \\
\hline 3.88 & $-2 \cdot 16$ & 4.28 & $-2 \cdot 40$ & 4.68 & -2.65 & 5.08 & -2.91 \\
\hline 3.89 & $-2 \cdot 16$ & 4.29 & $-2 \cdot 41$ & 4.69 & -2.66 & 5.09 & -2.91 \\
\hline 3.90 & -2.17 & 4.30 & $-2 \cdot 41$ & 4.70 & -2.66 & 5.10 & -2.92 \\
\hline 3.91 & -2.17 & 4.31 & $-2 \cdot 42$ & 4.71 & -2.67 & 5.11 & .93 \\
\hline 3.92 & -2.18 & 4.32 & $-2 \cdot 43$ & 4.72 & -2.68 & 5.12 & -2.93 \\
\hline 3.93 & -2.19 & 4.33 & $-2 \cdot 43$ & 4.73 & -2.68 & 5.13 & -2.94 \\
\hline 3.94 & -2.19 & 4.34 & -2.44 & 4.74 & -2.69 & 5.14 & -2.95 \\
\hline 3.95 & $-2 \cdot 20$ & 4.35 & -2.45 & 4.75 & -2.70 & 5.15 & -2.95 \\
\hline 3.96 & $-2 \cdot 20$ & 4.36 & -2.45 & 4.76 & -2.70 & 5.16 & -2.96 \\
\hline 3.97 & -2.21 & 4.37 & $-2 \cdot 46$ & 4.77 & -2.71 & 5.17 & -2.9 \\
\hline 3.98 & $-2 \cdot 22$ & 4.38 & $-2 \cdot 46$ & 4.78 & -2.72 & 5.18 & -2.9 \\
\hline 99 & $-2 \cdot 22$ & 4.39 & -2.47 & 4.79 & -2.72 & 5.19 & $-2 \cdot 9$ \\
\hline 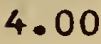 & -2.23 & 4.40 & -2.48 & 4.80 & -2.73 & 5.20 & -2.98 \\
\hline
\end{tabular}




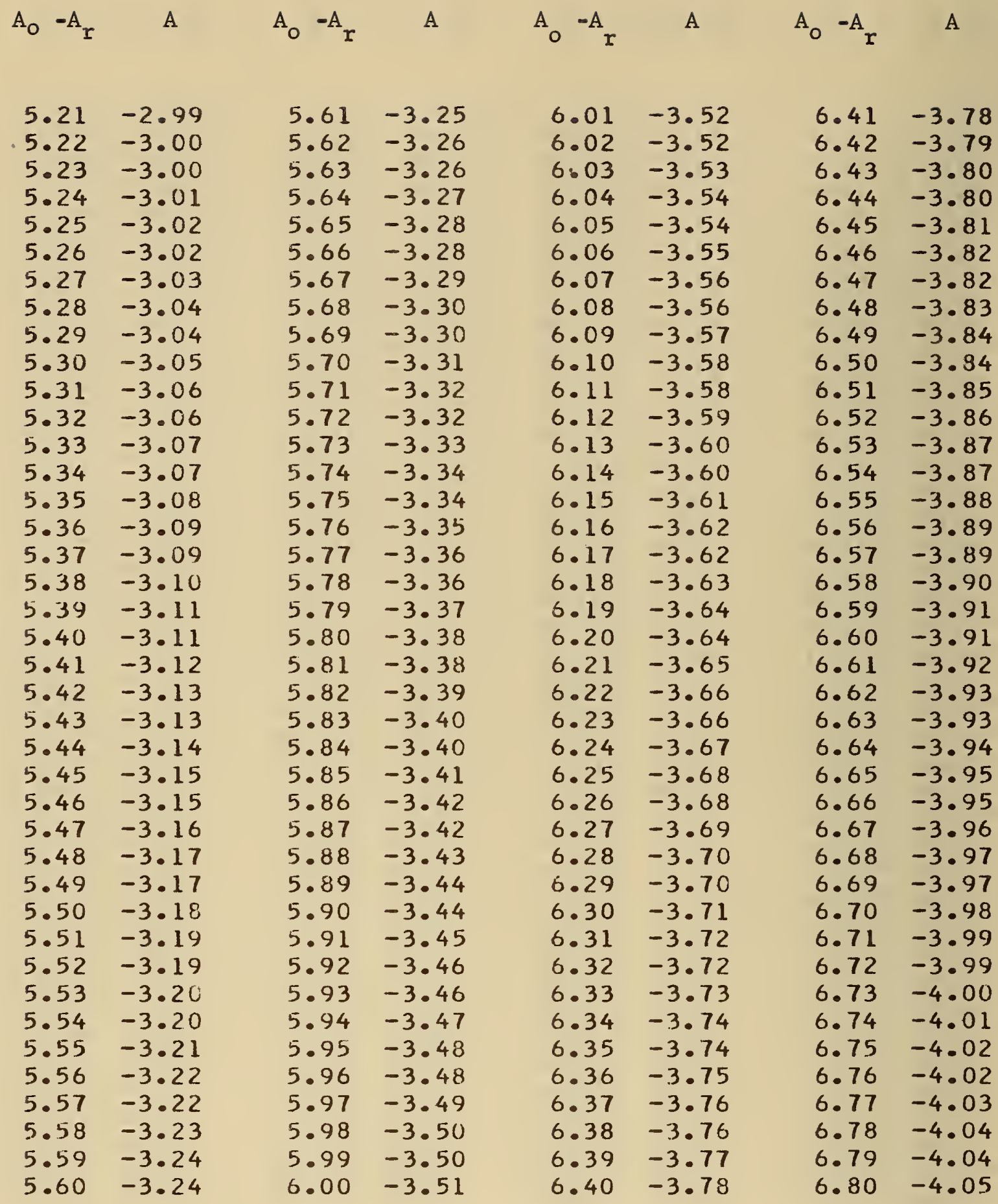




\begin{tabular}{|c|c|c|c|c|c|c|c|}
\hline 6.81 & -4.06 & 7.21 & $-4 \cdot 33$ & 7.61 & -4.61 & 3.01 & -4.90 \\
\hline 6.82 & -4.06 & 7.22 & $-4 \cdot 34$ & 7.62 & -4.62 & 8.02 & -4.91 \\
\hline 6.83 & -4.07 & 7.23 & $-4 \cdot 35$ & 7.63 & -4.63 & 8.03 & -4.91 \\
\hline 6.84 & -4.08 & 7.24 & $-4 \cdot 35$ & 7.64 & -4.63 & 8.04 & -4.92 \\
\hline 6.85 & -4.08 & 7.25 & $-4 \cdot 36$ & 7.65 & -4.64 & 8.05 & -4.93 \\
\hline 6.86 & -4.09 & 7.26 & $-4 \cdot 37$ & 7.66 & -4.65 & 8.06 & -4.93 \\
\hline 6.87 & $-4 \cdot 10$ & 7.27 & -4.37 & 7.67 & -4.66 & 8.07 & $-4 \cdot 94$ \\
\hline 6.88 & $-4 \cdot 10$ & 7.28 & -4.38 & 7.68 & -4.66 & 8.08 & -4.95 \\
\hline 6.89 & -4.11 & 7.29 & $-4 \cdot 39$ & 7.69 & -4.67 & 8.09 & $-4 \cdot 96$ \\
\hline 6.90 & -4.12 & $7 \cdot 30$ & -4.40 & 7.70 & -4.68 & 8.10 & -4.96 \\
\hline 6.91 & -4.13 & 7.31 & -4.40 & 7.71 & -4.68 & 3.11 & -4.97 \\
\hline 6.92 & -4.13 & 7.32 & -4.41 & 7.72 & -4.69 & 8.12 & -4.98 \\
\hline 6.93 & $-4 \cdot 14$ & 7.33 & -4.42 & 7.73 & -4.70 & 8.13 & -4.98 \\
\hline 6.94 & -4.15 & 7.34 & $-4 \cdot 42$ & 7.74 & -4.71 & 8.14 & -4.99 \\
\hline 6.95 & $-4 \cdot 15$ & 7.35 & $-4 \cdot 43$ & 7.75 & -4.71 & 8.15 & -5.00 \\
\hline 6.96 & -4.16 & $7 \cdot 36$ & $-4 \cdot 44$ & 7.76 & -4.72 & 8.16 & -5.01 \\
\hline 6.97 & -4.17 & 7.37 & -4.44 & 7.77 & -4.73 & 8.17 & -5.01 \\
\hline 6.98 & -4.17 & $7 \cdot 38$ & -4.45 & 7.78 & -4.73 & 8.18 & -5.02 \\
\hline 6.99 & -4.18 & 7.39 & $-4 \cdot 46$ & 7.79 & -4.74 & 8.19 & -5.03 \\
\hline 7.00 & -4.19 & 7.40 & -4.47 & 7.80 & -4.75 & 8.20 & -5.03 \\
\hline 7.01 & -4.19 & 7.41 & -4.47 & 7.81 & -4.76 & 8.21 & -5.04 \\
\hline 7.02 & $-4 \cdot 20$ & 7.42 & -4.48 & 7.82 & -4.76 & 8.22 & -5.05 \\
\hline 7.03 & $-4 \cdot 21$ & 7.43 & -4.49 & 7.83 & -4.77 & 8.23 & -5.06 \\
\hline 7.04 & -4.22 & 7.44 & $-4 \cdot 49$ & 7.84 & -4.78 & 8.24 & -5.06 \\
\hline 7.05 & -4.22 & 7.45 & $-4 \cdot 50$ & 7.85 & -4.78 & 8.25 & -5.07 \\
\hline 7.06 & $-4 \cdot 23$ & 7.46 & $-4 \cdot 51$ & 7.86 & -4.79 & 8.26 & $-5 \cdot 0$ \\
\hline 7.07 & -4.24 & 7.47 & -4.51 & 7.87 & -4.80 & 8.27 & -5.08 \\
\hline 7.08 & $-4 \cdot 24$ & 7.48 & $-4 \cdot 52$ & 7.88 & -4.81 & 8.28 & -5.09 \\
\hline 7.09 & $-4 \cdot 25$ & 7.49 & -4.53 & 7.89 & -4.81 & 8.29 & $-5 \cdot 10$ \\
\hline 7.10 & -4.26 & 7.50 & $-4 \cdot 54$ & 7.90 & -4.82 & 8.30 & -5.11 \\
\hline 7.11 & -4.26 & 7.51 & $-4 \cdot 54$ & 7.91 & -4.83 & 8.31 & $-5 \cdot 1$ \\
\hline 7.12 & -4.27 & 7.52 & $-4 \cdot 55$ & 7.92 & -4.83 & $8 \cdot 32$ & $-5 \cdot 1$ \\
\hline 7.13 & $-4 \cdot 28$ & 7.53 & -4.56 & 7.93 & -4.84 & 8.33 & -5.13 \\
\hline 7.14 & $-4 \cdot 28$ & 7.54 & -4.56 & 7.94 & -4.85 & 8.34 & $-5 \cdot 14$ \\
\hline 7.15 & -4.29 & 7.55 & -4.57 & 7.95 & -4.85 & 8.35 & $-5 \cdot 14$ \\
\hline 7.16 & $-4 \cdot 30$ & 7.56 & -4.58 & 7.96 & $-4 \cdot 86$ & 8.36 & -5.15 \\
\hline 7.17 & -4.31 & 7.57 & -4.59 & 7.97 & -4.87 & 8.37 & $-5 \cdot 16$ \\
\hline 7.18 & $-4 \cdot 31$ & 7.58 & $-4 \cdot 59$ & 7.98 & -4.88 & 8.38 & $-5 \cdot 1$ \\
\hline 7.19 & $-4 \cdot 32$ & 7.59 & -4.60 & 7.99 & -4.88 & 8.39 & $-5 \cdot 1$ \\
\hline 7.20 & $-4 \cdot 33$ & 7.60 & -4.61 & 8.00 & -4.89 & 8.40 & $-5 \cdot 1$ \\
\hline
\end{tabular}



$A_{0}-A_{r}$
A
$A_{O}-A_{r}$
A
$A_{0}-A_{r}$
A
$A_{0}-A_{r}$
A

$\begin{array}{llll}8.41 & -5.19 & 8.81 & -5.48 \\ 8.42 & -5.19 & 8.82 & -5.48 \\ 8.43 & -5.20 & 8.83 & -5.49 \\ 8.44 & -5.21 & 8.84 & -5.50 \\ 8.45 & -5.21 & 8.85 & -5.51 \\ 8.46 & -5.22 & 8.86 & -5.51 \\ 8.47 & -5.23 & 8.87 & -5.52 \\ 8.48 & -5.24 & 8.88 & -5.53 \\ 8.49 & -5.24 & 8.89 & -5.54 \\ 8.50 & -5.25 & 8.90 & -5.54 \\ 8.51 & -5.26 & 8.91 & -5.55 \\ 8.52 & -5.27 & 8.92 & -5.56 \\ 8.53 & -5.27 & 8.93 & -5.57 \\ 8.54 & -5.28 & 8.94 & -5.57 \\ 8.55 & -5.29 & 8.95 & -5.58 \\ 8.56 & -5.29 & 8.96 & -5.59 \\ 8.57 & -5.30 & 8.97 & -5.60 \\ 8.58 & -5.31 & 8.98 & -5.60 \\ 8.59 & -5.32 & 8.99 & -5.61 \\ 8.60 & -5.32 & 9.00 & -5.62 \\ 8.61 & -5.33 & 9.01 & -5.62 \\ 8.62 & -5.34 & 9.02 & -5.63 \\ 8.63 & -5.35 & 9.03 & -5.64 \\ 8.64 & -5.35 & 9.04 & -5.65 \\ 8.65 & -5.36 & 9.05 & -5.65 \\ 8.66 & -5.37 & 9.06 & -5.66 \\ 8.67 & -5.38 & 9.07 & -5.67 \\ 8.68 & -5.38 & 9.08 & -5.68 \\ 8.69 & -5.39 & 9.09 & -5.68 \\ 8.70 & -5.40 & 9.10 & -5.69 \\ 8.71 & -5.40 & 9.11 & -5.70 \\ 8.72 & -5.41 & 9.12 & -5.71 \\ 8.73 & -5.42 & 9.13 & -5.71 \\ 8.74 & -5.43 & 9.14 & -5.72 \\ 8.75 & -5.43 & 9.15 & -5.73 \\ 8.76 & -5.44 & 9.16 & -5.74 \\ 8.77 & -5.45 & 9.17 & -5.74 \\ 8.78 & -5.46 & 9.18 & -5.75 \\ 8.79 & -5.46 & 9.19 & -5.76 \\ 8.80 & -5.47 & 9.20 & -5.77\end{array}$

$\begin{array}{llll}9.21 & -5.77 & 9.61 & -6.07 \\ 9.22 & -5.78 & 9.62 & -6.08 \\ 9.23 & -5.79 & 9.63 & -6.09 \\ 9.24 & -5.80 & 9.64 & -6.09 \\ 9.25 & -5.80 & 9.65 & -6.10 \\ 9.26 & -5.81 & 9.66 & -6.11 \\ 9.27 & -5.82 & 9.67 & -6.12 \\ 9.28 & -5.83 & 9.68 & -6.12 \\ 9.29 & -5.83 & 9.69 & -6.13 \\ 9.30 & -5.84 & 9.70 & -6.14 \\ 9.31 & -5.85 & 9.71 & -6.15 \\ 9.32 & -5.85 & 9.72 & -6.15 \\ 9.33 & -5.86 & 9.73 & -6.16 \\ 9.34 & -5.87 & 9.74 & -6.17 \\ 9.35 & -5.88 & 9.75 & -6.18 \\ 9.36 & -5.88 & 9.76 & -6.18 \\ 9.37 & -5.89 & 9.77 & -6.19 \\ 9.38 & -5.90 & 9.78 & -6.20 \\ 9.39 & -5.91 & 9.79 & -6.21 \\ 9.40 & -5.91 & 9.80 & -6.21 \\ 9.41 & -5.92 & 9.81 & -6.22 \\ 9.42 & -5.93 & 9.82 & -6.23 \\ 9.43 & -5.94 & 9.83 & -6.24 \\ 9.44 & -5.94 & 9.84 & -6.25 \\ 9.45 & -5.95 & 9.85 & -6.25 \\ 9.46 & -5.96 & 9.86 & -6.26 \\ 9.47 & -5.97 & 9.87 & -6.27 \\ 9.48 & -5.97 & 9.88 & -6.28 \\ 9.49 & -5.98 & 9.89 & -6.28 \\ 9.50 & -5.99 & 9.90 & -6.29 \\ 9.51 & -6.00 & 9.91 & -6.30 \\ 9.52 & -6.00 & 9.92 & -6.31 \\ 9.53 & -6.01 & 9.93 & -6.31 \\ 9.54 & -6.02 & 9.94 & -6.32 \\ 9.55 & -6.03 & 9.95 & -6.33 \\ 9.56 & -6.03 & 9.96 & -6.34 \\ 9.57 & -6.04 & 9.97 & -6.34 \\ 9.58 & -6.05 & 9.98 & -6.35 \\ 9.59 & -6.06 & 9.99 & -6.36 \\ 9.60 & -6.06 & 10.00 & -6.37\end{array}$



$A_{0}-A_{r}$
A
$A_{0}-A_{r}$
A
$A_{0}-A_{r}$
A
$A_{0}-A_{r}$
A

\begin{tabular}{|c|c|c|c|c|c|c|c|}
\hline 10.01 & -6.37 & 10.41 & -6.68 & 10.81 & -6.99 & 11.21 & $-7 \cdot 30$ \\
\hline 10.02 & -6.38 & 10.42 & -6.69 & 10.82 & -7.00 & 11.22 & $-7 \cdot 31$ \\
\hline 10.03 & -6.39 & 10.43 & -6.70 & 10.83 & -7.00 & 11.23 & $-7 \cdot 32$ \\
\hline 10.04 & -6.40 & $10 \cdot 44$ & -6.70 & 10.84 & -7.01 & 11.24 & -7.32 \\
\hline 10.05 & -6.40 & 10.45 & -6.71 & 10.85 & -7.02 & 11.25 & $-7 \cdot 3$ \\
\hline 10.06 & $-6 \cdot 41$ & 10.46 & -6.72 & 10.86 & -7.03 & 11.26 & $-7 \cdot 34$ \\
\hline 10.07 & $-6 \cdot 42$ & 10.47 & -6.73 & 10.87 & -7.04 & 11.27 & -7.35 \\
\hline 10.08 & $-6 \cdot 43$ & 10.48 & -6.73 & 10.88 & -7.04 & 11.28 & $-7 \cdot 36$ \\
\hline 10.09 & -6.43 & 10.49 & -6.74 & 10.89 & -7.05 & 11.29 & $-7 \cdot 36$ \\
\hline 10.10 & -6.44 & 10.50 & -6.75 & 10.90 & -7.06 & 11.30 & -7.37 \\
\hline 10.11 & -6.45 & 10.51 & -6.76 & 10.91 & -7.07 & 11.31 & $-7 \cdot 3$ \\
\hline $10 \cdot 12$ & -6.46 & 10.52 & -6.76 & 10.92 & -7.07 & $11 \cdot 32$ & $-7 \cdot 3$ \\
\hline 10.13 & -6.47 & 10.53 & -6.77 & 10.93 & -7.08 & 11.33 & -7.4 \\
\hline 10.14 & -6.47 & 10.54 & -6.78 & 10.94 & -7.09 & 11.34 & $-7 \cdot 4$ \\
\hline 10.15 & $-6 \cdot 48$ & 10.55 & -6.79 & 10.95 & -7.10 & 11.35 & -7.4 \\
\hline 10.16 & -6.49 & 10.56 & -6.80 & 10.96 & -7.11 & 11.36 & $-7 \cdot 4$ \\
\hline 10.17 & -6.50 & 10.57 & -6.80 & 10.97 & -7.11 & 11.37 & $-7 \cdot 4$ \\
\hline 10.18 & $-6 \cdot 50$ & 10.58 & -6.81 & 10.98 & -7.12 & 11.38 & $-7 \cdot 4$ \\
\hline 10.19 & -6.51 & 10.59 & -6.82 & 10.99 & -7.13 & 11.39 & $-7 \cdot 4$ \\
\hline 10.20 & -6.52 & 10.60 & -6.83 & 11.00 & -7.14 & 11.40 & -7.4 \\
\hline 10.21 & -6.53 & 10.61 & -6.83 & 11.01 & $-7 \cdot 14$ & 11.41 & $-7 \cdot 4$ \\
\hline 10.22 & -6.53 & 10.62 & $-6 \cdot 84$ & 11.02 & -7.15 & 11.42 & $-7 \cdot 4$ \\
\hline 10.23 & $-6 \cdot 54$ & 10.63 & -6.85 & 11.03 & -7.16 & 11.43 & -7.4 \\
\hline 10.24 & -6.55 & 10.64 & -6.86 & 11.04 & -7.17 & 11.44 & $-7 \cdot 4$ \\
\hline 10.25 & -6.56 & 10.65 & -6.86 & 11.05 & -7.18 & 11.45 & $-7 \cdot 4$ \\
\hline 10.26 & -6.56 & 10.66 & -6.87 & 11.06 & -7.18 & 11.46 & -7.5 \\
\hline 10.27 & -6.57 & 10.67 & -6.88 & 11.07 & -7.19 & 11.47 & -7.5 \\
\hline $10 \cdot 28$ & -6.58 & 10.68 & -6.89 & 11.08 & -7.20 & 11.48 & $-7 \cdot 5$ \\
\hline 10.29 & -6.59 & 10.69 & -6.90 & 11.09 & -7.21 & 11.49 & $-7 \cdot 5$ \\
\hline 10.30 & -6.60 & 10.70 & -6.90 & 11.10 & $-7 \cdot 21$ & 11.50 & $-7 \cdot 5$ \\
\hline 10.31 & -6.60 & 10.71 & -6.91 & 11.11 & -7.22 & 11.51 & -7.5 \\
\hline 10.32 & -6.61 & 10.72 & -6.92 & 11.12 & -7.23 & 11.52 & -7.5 \\
\hline 10.33 & -6.62 & 10.73 & -6.93 & 11.13 & -7.24 & 11.53 & $-7 \cdot 5$ \\
\hline 10.34 & -6.63 & 10.74 & -6.93 & 11.14 & -7.25 & 11.54 & $-7 \cdot 5$ \\
\hline 10.35 & -6.63 & 10.75 & -6.94 & 11.15 & -7.25 & 11.55 & -7.5 \\
\hline $10 \cdot 36$ & -6.64 & 10.76 & -6.95 & $11 \cdot 16$ & -7.26 & 11.56 & $-7 \cdot 5$ \\
\hline 10.37 & -6.65 & 10.77 & -6.96 & 11.17 & -7.27 & 11.57 & -7.5 \\
\hline 10.38 & -6.66 & 10.78 & -6.97 & 11.18 & -7.28 & 11.58 & $-7 \cdot 5$ \\
\hline 10.39 & -6.66 & 10.79 & -6.97 & 11.19 & -7.29 & 11.59 & -7.6 \\
\hline 10.40 & -6.67 & 10.80 & -6.98 & 11.20 & -7.29 & 11.60 & $-7 \cdot 6$ \\
\hline
\end{tabular}




\begin{tabular}{|c|c|c|c|c|c|c|c|}
\hline 11.61 & -7.62 & 12.01 & -7.93 & $12 \cdot 41$ & -8.26 & 12.81 & -8.58 \\
\hline 11.62 & -7.62 & 12.02 & -7.94 & $12 \cdot 42$ & $-8 \cdot 26$ & 12.82 & -8.59 \\
\hline 11.63 & -7.63 & 12.03 & -7.95 & 12.43 & -8.27 & 12.83 & $-8 \cdot 6 c$ \\
\hline 11.64 & -7.64 & 12.04 & -7.96 & $12 \cdot 44$ & $-8 \cdot 28$ & 12.84 & -8.6 \\
\hline 11.65 & -7.65 & 12.05 & -7.97 & 12.45 & -8.29 & 12.85 & $-8 \cdot 6$ \\
\hline 11.66 & -7.66 & 12.06 & -7.97 & 12.46 & $-8 \cdot 30$ & 12.86 & -8.6 \\
\hline 1.67 & -7.66 & 12.07 & -7.98 & 12.47 & -8.30 & 12.87 & -8.6 \\
\hline 1.68 & -7.67 & 12.08 & -7.99 & $12 \cdot 48$ & -8.31 & 12.88 & $-8 \cdot 6$ \\
\hline 1.69 & -7.68 & 12.09 & -8.00 & 12.49 & $-8 \cdot 32$ & 12.89 & $-8 \cdot 6$ \\
\hline 1.70 & -7.69 & $12 \cdot 10$ & -8.01 & $12 \cdot 50$ & -8.33 & 12.90 & -8.6 \\
\hline 1.71 & -7.70 & $12 \cdot 11$ & -8.01 & 12.51 & $-8 \cdot 34$ & 12.91 & $-8 \cdot 6$ \\
\hline 1.72 & -7.70 & 12.12 & -8.02 & 12.52 & $-8 \cdot 34$ & 12.92 & $-8 \cdot 6$ \\
\hline 1.73 & -7.71 & 12.13 & -8.03 & 12.53 & -8.35 & 12.93 & -8.6 \\
\hline 1.74 & -7.72 & $12 \cdot 14$ & -8.04 & 12.54 & -8.36 & 12.94 & -8.6 \\
\hline 1.75 & -7.73 & $12 \cdot 15$ & -8.05 & 12.55 & -8.37 & 12.95 & -8.6 \\
\hline 1.76 & -7.74 & $12 \cdot 16$ & -8.05 & 12.56 & -8.38 & 12.96 & -8.7 \\
\hline 1.77 & -7.74 & 12.17 & -8.06 & 12.57 & -8.38 & 12.97 & -8.7 \\
\hline 1.78 & -7.75 & 12.18 & -8.07 & 12.58 & -8.39 & 12.98 & -8.7 \\
\hline 1.79 & -7.76 & 12.19 & -8.08 & 12.59 & $-8 \cdot 40$ & 12.99 & -8.7 \\
\hline 1.80 & -7.77 & 12.20 & -8.09 & 12.60 & -8.41 & 13.00 & -8.7 \\
\hline 11.81 & -7.77 & $12 \cdot 21$ & -8.09 & 12.61 & $-8 \cdot 42$ & 13.01 & -8.7 \\
\hline 11.82 & -7.78 & 12.22 & -8.10 & 12.62 & -8.43 & 13.02 & -8.7 \\
\hline 1.83 & -7.79 & 12.23 & -8.11 & 12.63 & -8.43 & 13.03 & -8.7 \\
\hline 11.84 & -7.80 & $12 \cdot 24$ & $-8 \cdot 12$ & 12.64 & -8.44 & 13.04 & -8.7 \\
\hline 11.85 & -7.81 & 12.25 & -8.13 & 12.65 & -8.45 & 13.05 & -8.7 \\
\hline 1.86 & -7.81 & $12 \cdot 26$ & -8.13 & 12.66 & -8.46 & 13.06 & -8.7 \\
\hline 1.87 & -7.82 & 12.27 & -8.14 & 12.67 & -8.47 & 13.07 & -8.7 \\
\hline 1.88 & -7.83 & 12.28 & $-8 \cdot 15$ & 12.68 & -8.47 & 13.08 & -8.8 \\
\hline 1.89 & -7.84 & 12.29 & $-8 \cdot 16$ & 12.69 & -8.48 & 13.09 & -8.8 \\
\hline 1.90 & -7.85 & $12 \cdot 30$ & -8.17 & 12.70 & -8.49 & 13.10 & -8.8 \\
\hline 1.91 & -7.85 & 12.31 & -8.17 & 12.71 & $-8 \cdot 50$ & 13.11 & -8.8 \\
\hline 1.92 & -7.86 & 12.32 & -8.18 & 12.72 & -8.51 & 13.12 & -8.8 \\
\hline 11.93 & -7.87 & 12.33 & -8.19 & 12.73 & -8.51 & 13.13 & -8.8 \\
\hline 1.94 & -7.88 & $12 \cdot 34$ & $-8 \cdot 20$ & 12.74 & -8.52 & 13.14 & -8.8 \\
\hline 11.95 & -7.89 & $12 \cdot 35$ & $-8 \cdot 21$ & 12.75 & -8.53 & 13.15 & $-8 \cdot 8$ \\
\hline 11.96 & -7.89 & 12.36 & $-8 \cdot 22$ & 12.76 & -8.54 & 13.16 & -8.8 \\
\hline 11.97 & -7.90 & 12.37 & $-8 \cdot 22$ & 12.77 & -8.55 & 13.17 & $-8 \cdot 8$ \\
\hline 11.98 & -7.91 & $12 \cdot 38$ & -8.23 & 12.78 & -8.56 & 13.18 & -8.8 \\
\hline 1.99 & -7.92 & 12.39 & $-8 \cdot 24$ & 12.79 & -8.56 & 13.19 & -8.8 \\
\hline 2.00 & -7.93 & 12.40 & -8.25 & 12.80 & -8.57 & 13.20 & -8.9 \\
\hline
\end{tabular}



$A_{0}-A_{r} \quad A$
$A_{0}-A_{r}$
A
$A_{0}-A_{I}$
A
$A_{0}-A_{r}$
A

\begin{tabular}{|c|c|c|c|c|c|c|c|}
\hline 13.21 & -8.91 & 13.61 & -9.24 & 14.01 & -9.57 & 14.41 & -9.90 \\
\hline 13.22 & -8.91 & 13.62 & -9.24 & 14.02 & -9.58 & 14.42 & -9.91 \\
\hline 13.23 & -8.92 & 13.63 & -9.25 & 14.03 & -9.59 & 14.43 & -9.92 \\
\hline 13.24 & -8.93 & 13.64 & -9.26 & 14.04 & -9.59 & 14.44 & -9.93 \\
\hline 13.25 & -8.94 & 13.65 & -9.27 & 14.05 & -9.60 & 14.45 & -9.94 \\
\hline 13.26 & -8.95 & 13.66 & -9.28 & 14.06 & -9.61 & 14.46 & -9.95 \\
\hline 13.27 & -8.96 & 13.67 & -9.29 & 14.07 & -9.62 & 14.47 & -9.95 \\
\hline $13 \cdot 28$ & -8.96 & 13.68 & -9.29 & 14.08 & -9.63 & 14.48 & -9.96 \\
\hline 13.29 & -8.97 & 13.69 & -9.30 & 14.09 & -9.64 & 14.49 & -9.97 \\
\hline 13.30 & -8.98 & 13.70 & -9.31 & 14.10 & -9.64 & 14.50 & -9.98 \\
\hline 31 & -8.99 & 13.71 & -9.32 & 14.11 & -9.65 & 14.51 & -9.9 \\
\hline 13.32 & -9.00 & 13.72 & -9.33 & 14.12 & -9.66 & 14.52 & -10.00 \\
\hline 33 & -9.01 & 13.73 & -9.34 & 14.13 & -9.67 & 14.53 & -10.00 \\
\hline 13.34 & -9.01 & 13.74 & -9.34 & 14.14 & -9.68 & 14.54 & -10.01 \\
\hline 35 & -9.02 & 13.75 & -9.35 & 14.15 & -9.69 & 14.55 & -10.02 \\
\hline 36 & -9.03 & 13.76 & -9.36 & 14.16 & -9.69 & 14.56 & -10.03 \\
\hline 37 & -9.04 & 13.77 & -9.37 & 14.17 & -9.70 & 14.57 & -10.04 \\
\hline 38 & -9.05 & 13.78 & -9.38 & 14.18 & -9.71 & 14.58 & -10.0 \\
\hline 39 & -9.05 & 13.79 & -9.39 & 14.19 & -9.72 & 14.59 & -10.05 \\
\hline 13.40 & -9.06 & 13.80 & -9.39 & 14.20 & -9.73 & 14.60 & -10.06 \\
\hline 41 & -9.07 & 13.81 & -9.40 & 14.21 & -9.74 & 14.61 & -10.0 \\
\hline 42 & -9.08 & 13.82 & -9.41 & 14.22 & -9.74 & 14.62 & -10.08 \\
\hline 43 & -9.09 & 13.83 & -9.42 & 14.23 & -9.75 & 14.63 & -10.09 \\
\hline 44 & -9.10 & 13.84 & -9.43 & $14 \cdot 24$ & -9.76 & 14.64 & $-10 \cdot 10$ \\
\hline 45 & -9.10 & 13.85 & -9.44 & 14.25 & -9.77 & 14.65 & $-10 \cdot 11$ \\
\hline 46 & -9.11 & 13.86 & -9.44 & 14.26 & -9.78 & 14.66 & -10.1 \\
\hline 47 & -9.12 & 13.87 & -9.45 & 14.27 & -9.79 & 14.67 & $-10 \cdot 12$ \\
\hline 48 & -9.13 & 13.88 & -9.46 & 14.28 & -9.79 & 14.68 & -10.13 \\
\hline 49 & -9.14 & 13.89 & -9.47 & 14.29 & -9.80 & 14.69 & -10.1 \\
\hline 50 & -9.15 & 13.90 & -9.48 & 14.30 & -9.81 & 14.70 & $-10 \cdot 15$ \\
\hline 51 & -9.15 & 13.91 & -9.49 & 14.31 & -9.82 & 14.71 & $-10 \cdot 1$ \\
\hline 52 & -9.16 & 13.92 & -9.49 & 14.32 & -9.83 & 14.72 & -10.1 \\
\hline 53 & -9.17 & 13.93 & -9.50 & 14.33 & -9.84 & 14.73 & -10.17 \\
\hline 54 & -9.18 & 13.94 & -9.51 & 14.34 & -9.84 & 14.74 & $-10 \cdot 1$ \\
\hline 55 & -9.19 & 13.95 & -9.52 & 14.35 & -9.85 & 14.75 & $-10 \cdot 1$ \\
\hline 56 & -9.19 & 13.96 & -9.53 & 14.36 & -9.86 & 14.76 & $-10 \cdot 2$ \\
\hline 57 & -9.20 & 13.97 & -9.54 & 14.37 & -9.87 & 14.77 & -10.2 \\
\hline 58 & -9.21 & 13.98 & -9.54 & 14.38 & -9.88 & 14.78 & $-10 \cdot 2$ \\
\hline & -9.22 & 13.99 & -9.55 & 14.39 & -9.89 & 14.79 & -10. \\
\hline & -9.23 & 14.00 & -9.56 & 14.40 & -9.89 & 14.80 & 10 \\
\hline
\end{tabular}


$A_{0}-A_{r} \quad A$

$14.81-10.24$

$14.82-10.25$

$14.83-10.26$

$14.84-10.27$

$14.85-10.27$

$14.86-10.28$

$14.87-10.29$

$14.88-10.30$

$14.89-10.31$

$14.90-10.32$

$14.91-10.33$

$14.92-10.33$

$14.93-10.34$

$14.94-10.35$

$14.95-10.36$

$14.96-10.37$

$14.97-10.38$

$14.98-10.38$

$14.99-10.39$

$15.00-10.40$

$15.01-10.41$

$15.02-10.42$

$15.13-10.43$

$15.04-10.44$

$15.05-10.44$

$15.06-10.45$

$15.07-10.46$

$15.08-10.47$

$15.09-10.48$

$15.10-10.49$

$15.11-10.49$

$15.12-10.50$

$15.13-10.51$

$15.14-10.52$

$15.15-10.53$

$15.16-10.54$

$15.17-10.55$

$15.18-10.55$

$15.19-10.56$

$15.20-10.57$
$A_{0}-A_{r}$

A

$A_{0}-A_{r}$

A

$A_{0}-A_{r}$

A

$\begin{array}{llll}15.21-10.58 & 15.61-10.92 & 16.01-11.27 \\ 15.22-10.59 & 15.62-10.93 & 16.02-11.27 \\ 15.23-10.60 & 15.63-10.94 & 16.03-11.28 \\ 15.24-10.61 & 15.64-10.95 & 16.04-11.29 \\ 15.25-10.61 & 15.65-10.96 & 16.05-11.30 \\ 15.26-10.62 & 15.66-10.96 & 16.06-11.31 \\ 15.27-10.63 & 15.67-10.97 & 16.07-11.32 \\ 15.28-10.64 & 15.68-10.98 & 16.08-11.33 \\ 15.29-10.65 & 15.69-10.99 & 16.09-11.34 \\ 15.30-10.66 & 15.70-11.00 & 16.10-11.34 \\ 15.31-10.67 & 15.71-11.01 & 16.11-11.35 \\ 15.32-10.67 & 15.72-11.02 & 16.12-11.36 \\ 15.33-10.68 & 15.73-11.03 & 16.13-11.37 \\ 15.34-10.69 & 15.74-11.03 & 16.14-11.38 \\ 15.35-10.70 & 15.75-11.04 & 16.15-11.39 \\ 15.36-10.71 & 15.76-11.05 & 16.16-11.40 \\ 15.37-10.72 & 15.77-11.06 & 16.17-11.40 \\ 15.38-10.73 & 15.78-11.07 & 16.18-11.41 \\ 15.39-10.73 & 15.79-11.08 & 16.19-11.42 \\ 15.40-10.74 & 15.80-11.09 & 16.20-11.43 \\ 15.41-10.75 & 15.81-11.09 & 16.21-11.44 \\ 15.42-10.76 & 15.82-11.10 & 16.22-11.45 \\ 15.43-10.77 & 15.83-11.11 & 16.23-11.46 \\ 15.44-10.78 & 15.84-11.12 & 16.24-11.47 \\ 15.45-10.78 & 15.85-11.13 & 16.25-11.47 \\ 15.46-10.79 & 15.86-11.14 & 16.26-11.48 \\ 15.47-10.80 & 15.87-11.15 & 16.27-11.49 \\ 15.48-10.81 & 15.88-11.15 & 16.28-11.50 \\ 15.49-10.82 & 15.89-11.16 & 16.29-11.51 \\ 15.50-10.83 & 15.90-11.17 & 16.30-11.52 \\ 15.51-10.84 & 15.91-11.18 & 16.31-11.53 \\ 15.52-10.84 & 15.92-11.19 & 16.32-11.53 \\ 15.53-10.85 & 15.93-11.20 & 16.33-11.54 \\ 15.54-10.86 & 15.94-11.21 & 16.34-11.55 \\ 15.55-10.87 & 15.95-11.21 & 16.35-11.56 \\ 15.56-10.88 & 15.96-11.22 & 16.36-11.57 \\ 15.57-10.89 & 15.97-11.23 & 16.37-11.58 \\ 15.58-10.90 & 15.98-11.24 & 16.38-11.59 \\ 15.59-10.90 & 15.99-11.25 & 16.39-11.60 \\ 15.60-10.91 & 16.00-11.26 & 16.40-11.60\end{array}$




\begin{tabular}{|c|c|c|c|c|c|c|c|}
\hline$A_{0}-A_{r}$ & A & $A_{0}-A_{r}$ & A & $A_{0}{ }^{-A_{r}}$ & A & $A_{0}{ }^{-A_{r}}$ & $\mathrm{~A}$ \\
\hline 16.41 & -11.61 & 16.81 & -11.96 & 17.21 & $-12 \cdot 31$ & 17.61 & -12.66 \\
\hline 16.42 & -11.62 & 16.82 & -11.97 & 17.22 & $-12 \cdot 32$ & 17.62 & -12.67 \\
\hline 16.43 & -11.63 & 16.83 & $-11 \cdot 98$ & 17.23 & $-12 \cdot 33$ & 17.63 & $-12 \cdot 68$ \\
\hline 16.44 & -11.64 & 16.84 & -11.99 & 17.24 & $-12 \cdot 34$ & 17.64 & -12.69 \\
\hline 16.45 & -11.65 & 16.85 & -12.00 & 17.25 & $-12 \cdot 35$ & 17.65 & $-12 \cdot 70$ \\
\hline $16 \cdot 46$ & $-11 \cdot 66$ & 16.86 & -12.00 & 17.26 & $-12 \cdot 36$ & 17.66 & -12.71 \\
\hline 16.47 & -11.66 & 16.87 & $-12 \cdot 01$ & 17.27 & $-12 \cdot 36$ & 17.67 & $-12 \cdot 72$ \\
\hline 16.48 & -11.67 & 16.88 & $-12 \cdot 02$ & 17.28 & -12.37 & 17.68 & $-12 \cdot 73$ \\
\hline 16.49 & $-11 \cdot 68$ & 16.89 & -12.03 & 17.29 & $-12 \cdot 38$ & 17.69 & $-12 \cdot 73$ \\
\hline 16.50 & -11.69 & 16.90 & $-12 \cdot 04$ & $17 \cdot 30$ & $-12 \cdot 39$ & 17.70 & $-12 \cdot 74$ \\
\hline 16.51 & $-11 \cdot 70$ & 16.91 & -12.05 & 17.31 & $-12 \cdot 40$ & 17.71 & $-12 \cdot 75$ \\
\hline 16.52 & -11.71 & 16.92 & -12.06 & 17.32 & $-12 \cdot 41$ & 17.72 & $-12 \cdot 76$ \\
\hline 16.53 & -11.72 & 16.93 & -12.07 & 17.33 & $-12 \cdot 42$ & 17.73 & -12.77 \\
\hline 16.54 & $-11 \cdot 73$ & 16.94 & -12.07 & $17 \cdot 34$ & $-12 \cdot 43$ & 17.74 & $-12 \cdot 78$ \\
\hline 16.55 & -11.73 & 16.95 & $-12 \cdot 08$ & 17.35 & $-12 \cdot 43$ & 17.75 & -12.79 \\
\hline 16.56 & -11.74 & 16.96 & -12.09 & 17.36 & $-12 \cdot 44$ & 17.76 & $-12 \cdot 80$ \\
\hline 16.57 & -11.75 & 16.97 & $-12 \cdot 10$ & 17.37 & $-12 \cdot 45$ & 17.77 & $-12 \cdot 81$ \\
\hline 16.58 & -11.76 & 16.98 & -12.11 & 17.38 & $-12 \cdot 46$ & 17.78 & $-12 \cdot 81$ \\
\hline 16.59 & -11.77 & 16.99 & $-12 \cdot 12$ & 17.39 & $-12 \cdot 47$ & 17.79 & $-12 \cdot 82$ \\
\hline 16.60 & -11.78 & 17.00 & $-12 \cdot 13$ & 17.40 & $-12 \cdot 48$ & 17.80 & $-12 \cdot 83$ \\
\hline 16.61 & -11.79 & 17.01 & $-12 \cdot 14$ & 17.41 & $-12 \cdot 49$ & 17.81 & $-12 \cdot 84$ \\
\hline 16.62 & -11.80 & 17.02 & $-12 \cdot 14$ & 17.42 & $-12 \cdot 50$ & 17.82 & $-12 \cdot 85$ \\
\hline 16.63 & $-11 \cdot 80$ & 17.03 & $-12 \cdot 15$ & 17.43 & $-12 \cdot 51$ & 17.83 & $-12 \cdot 86$ \\
\hline 16.64 & -11.81 & 17.04 & $-12 \cdot 16$ & $17 \cdot 44$ & $-12 \cdot 51$ & 17.84 & $-12 \cdot 87$ \\
\hline 16.65 & -11.82 & 17.05 & $-12 \cdot 17$ & 17.45 & $-12 \cdot 52$ & 17.85 & $-12 \cdot 88$ \\
\hline 16.66 & -11.83 & 17.06 & $-12 \cdot 18$ & 17.46 & $-12 \cdot 53$ & 17.86 & $-12 \cdot 89$ \\
\hline 16.67 & -11.84 & 17.07 & $-12 \cdot 19$ & 17.47 & $-12 \cdot 54$ & 17.87 & $-12 \cdot 89$ \\
\hline 16.68 & -11.85 & 17.08 & $-12 \cdot 20$ & 17.48 & $-12 \cdot 55$ & 17.88 & $-12 \cdot 90$ \\
\hline 16.69 & $-11 \cdot 86$ & 17.09 & $-12 \cdot 21$ & 17.49 & $-12 \cdot 56$ & 17.89 & $-12 \cdot 91$ \\
\hline 16.70 & -11.87 & 17.10 & $-12 \cdot 22$ & 17.50 & -12.57 & 17.90 & $-12 \cdot 92$ \\
\hline 16.71 & -11.87 & 17.11 & $-12 \cdot 22$ & 17.51 & $-12 \cdot 58$ & $17 \cdot 91$ & $-12 \cdot 93$ \\
\hline 16.72 & -11.88 & 17.12 & $-12 \cdot 23$ & 17.52 & $-12 \cdot 58$ & 17.92 & $-12 \cdot 94$ \\
\hline 16.73 & -11.89 & 17.13 & $-12 \cdot 24$ & 17.53 & $-12 \cdot 59$ & 17.93 & $-12 \cdot 95$ \\
\hline 16.74 & $-11 \cdot 90$ & 17.14 & $-12 \cdot 25$ & 17.54 & $-12 \cdot 60$ & 17.94 & $-12 \cdot 96$ \\
\hline 16.75 & $-11 \cdot 91$ & 17.15 & $-12 \cdot 26$ & 17.55 & $-12 \cdot 61$ & 17.95 & $-12 \cdot 97$ \\
\hline 16.76 & -11.92 & 17.16 & $-12 \cdot 27$ & 17.56 & $-12 \cdot 62$ & 17.96 & $-12 \cdot 97$ \\
\hline 16.77 & -11.93 & 17.17 & $-12 \cdot 28$ & 17.57 & $-12 \cdot 63$ & 17.97 & $-12 \cdot 98$ \\
\hline 16.78 & -11.93 & 17.18 & $-12 \cdot 29$ & 17.58 & $-12 \cdot 64$ & 17.98 & $-12 \cdot 99$ \\
\hline 16.79 & $-11 \cdot 94$ & 17.19 & $-12 \cdot 29$ & 17.59 & -12.65 & 17.99 & -13.00 \\
\hline 16.80 & -11.95 & 17.20 & $-12 \cdot 30$ & 17.60 & $-12 \cdot 66$ & 18.00 & -13.01 \\
\hline
\end{tabular}




\begin{tabular}{|c|c|c|c|c|c|c|c|}
\hline $\mathrm{H}_{0}-\mathrm{A}_{\mathrm{r}}$ & A & $A_{0}-A_{I}$ & A & $A_{0}-A_{r}$ & A & $A_{0}-A_{r}$ & A \\
\hline 01 & -13.02 & $18 \cdot 41$ & $-13 \cdot 37$ & 18.81 & -13.73 & 19.21 & -14.09 \\
\hline 3.02 & -13.03 & 18.42 & -13.38 & 18.82 & -13.74 & 19.22 & -14.10 \\
\hline .03 & -13.04 & 16.43 & -13.39 & 18.83 & -13.75 & 19.23 & -14.11 \\
\hline 3.04 & -13.05 & 18.44 & $-13 \cdot 40$ & 18.84 & -13.76 & 19.24 & $-14 \cdot 12$ \\
\hline 25 & -13.05 & 18.45 & $-13 \cdot 41$ & 18.85 & -13.77 & 19.25 & -14.13 \\
\hline .06 & -13.06 & 18.46 & -13.42 & 18.86 & -13.78 & 19.26 & $-14 \cdot 14$ \\
\hline .07 & -13.07 & 18.47 & $-13 \cdot 43$ & 18.87 & -13.79 & 19.27 & -14.15 \\
\hline 3.08 & -13.08 & 18.48 & $-13 \cdot 44$ & 18.88 & -13.80 & 19.28 & -14.16 \\
\hline .09 & -13.09 & 18.49 & $-13 \cdot 45$ & 18.89 & -13.30 & 19.29 & $-14 \cdot 16$ \\
\hline & $-13 \cdot 10$ & 18.50 & $-13 \cdot 46$ & 18.90 & -13.81 & 19.30 & -14.17 \\
\hline 11 & -13.11 & 18.51 & -13.46 & 18.91 & -13.82 & 19.31 & -14.18 \\
\hline 12 & -13.12 & 18.52 & -13.47 & 18.92 & -13.83 & 19.32 & -14.19 \\
\hline & -13.13 & 18.53 & $-13 \cdot 48$ & 18.93 & $-13 \cdot 34$ & 19.33 & -14.20 \\
\hline 14 & -13.13 & 18.54 & -13.49 & 18.94 & -13.85 & 19.34 & $-14 \cdot 21$ \\
\hline 15 & $-13 \cdot 14$ & 1 b. 55 & -13.50 & 18.95 & -13.86 & 19.35 & -14.22 \\
\hline & $-13 \cdot 15$ & 18.36 & $-13 \cdot 51$ & 18.96 & -13.87 & 19.36 & -14.23 \\
\hline 17 & $-13 \cdot 16$ & 18.57 & $-13 \cdot 52$ & 18.97 & -13.88 & 19.37 & $-14 \cdot 24$ \\
\hline & -13.17 & 18.58 & -13.53 & 18.93 & -13.89 & 19.38 & -14.25 \\
\hline 19 & -13.18 & 18.59 & $-13 \cdot 54$ & 18.99 & -13.89 & 19.39 & -14.25 \\
\hline 20 & -13.19 & 18.60 & $-13 \cdot 54$ & 19.00 & -13.90 & 19.40 & -14.26 \\
\hline & $-13 \cdot 20$ & 18.61 & -13.55 & 19.01 & -13.91 & 19.41 & -14.27 \\
\hline 22 & -13.21 & 18.62 & -13.56 & 19.02 & $-13 \cdot 92$ & 19.42 & $-14 \cdot 28$ \\
\hline & -13.21 & 18.63 & -13.57 & 19.03 & $-13 \cdot 93$ & 19.43 & -14.29 \\
\hline .24 & $-13 \cdot 22$ & 18.64 & -13.58 & 19.04 & -13.94 & 19.44 & $-14 \cdot 30$ \\
\hline 25 & $-13 \cdot 23$ & 18.65 & -13.59 & 19.05 & -13.95 & 19.45 & $-14 \cdot 31$ \\
\hline & $-13 \cdot 24$ & 18.66 & -13.60 & 19.06 & -13.96 & 19.46 & $-14 \cdot 32$ \\
\hline 27 & -13.25 & 18.67 & -13.61 & 19.07 & -13.97 & 19.47 & $-14 \cdot 33$ \\
\hline & $-13 \cdot 26$ & 18.68 & -13.62 & 19.08 & $-13 \cdot 98$ & 19.48 & $-14 \cdot 34$ \\
\hline 29 & -13.27 & 18.69 & -13.63 & 19.09 & $-13 \cdot 98$ & 19.49 & -14.35 \\
\hline 30 & -13.28 & 18.70 & -13.63 & 19.10 & -13.99 & 19.50 & -14.35 \\
\hline & -13.29 & 18.71 & $-13 \cdot 64$ & 19.11 & -14.00 & 19.51 & $-14 \cdot 36$ \\
\hline & -13.29 & 18.72 & -13.65 & 19.12 & -14.01 & 19.52 & -14.37 \\
\hline & $-13 \cdot 30$ & 18.73 & $-13 \cdot 66$ & 19.13 & -14.02 & 19.53 & -14.38 \\
\hline & $-13 \cdot 31$ & 18.74 & -13.67 & 19.14 & -14.03 & 19.54 & -14.39 \\
\hline. & -13.32 & 18.75 & $-13 \cdot 68$ & 19.15 & -14.04 & 19.55 & $-14 \cdot 40$ \\
\hline & $-13 \cdot 33$ & 18.76 & -13.69 & 19.16 & -14.05 & 19.56 & $-14 \cdot 41$ \\
\hline & $-13 \cdot 34$ & 18.77 & $-13 \cdot 70$ & 19.17 & -14.06 & 19.57 & $-14 \cdot 42$ \\
\hline & -13.35 & 18.78 & -13.71 & 19.18 & -14.07 & 19.58 & $-14 \cdot 43$ \\
\hline & $-13 \cdot 36$ & 18.79 & -13.71 & 19.19 & -14.07 & 19.59 & $-14 \cdot 44$ \\
\hline & -13.37 & 18.80 & -13.72 & 19.20 & -14.08 & 19.60 & -14.44 \\
\hline
\end{tabular}




\begin{tabular}{|c|c|c|c|c|c|c|c|}
\hline$o^{-A_{r}}$ & A & $A_{0}-A_{r}$ & A & $A_{0}-A_{r}$ & A & $A_{0}-A_{r}$ & A \\
\hline 9.61 & $-14 \cdot 45$ & 20.01 & $-14 \cdot 32$ & 20.41 & $-15 \cdot 18$ & 20.81 & -15.55 \\
\hline 9.62 & -14.46 & 20.02 & -14.83 & $20 \cdot 42$ & -15.19 & 20.82 & -15.56 \\
\hline .63 & -14.47 & 20.03 & -14.83 & 20.43 & -15.20 & 20.83 & -15.57 \\
\hline .64 & $-14 \cdot 48$ & 20.04 & -14.84 & 20.44 & -15.21 & 20.84 & -15.57 \\
\hline .65 & -14.49 & 20.05 & -14.85 & 20.45 & $-15 \cdot 22$ & 20.85 & $-15 \cdot 58$ \\
\hline .66 & -14.50 & 20.06 & -14.86 & 20.46 & $-15 \cdot 23$ & 20.86 & -15.59 \\
\hline .67 & -14.51 & 20.07 & -14.87 & 20.47 & -15.24 & 20.87 & -15.60 \\
\hline .68 & $-14 \cdot 52$ & 20.08 & -14.88 & 20.48 & -15.25 & 20.88 & -15.61 \\
\hline 69 & -14.53 & 20.09 & -14.89 & 20.49 & -15.25 & 20.89 & -15.62 \\
\hline .70 & $-14 \cdot 54$ & 20.10 & $-14 \cdot 90$ & 20.50 & -15.26 & 20.90 & -15.63 \\
\hline .71 & -14.54 & 20.11 & -14.91 & 20.51 & -15.27 & 20.91 & $-15 \cdot 64$ \\
\hline & -14.55 & 20.12 & -14.92 & 20.52 & -15.28 & 20.92 & -15.65 \\
\hline .73 & -14.56 & 20.13 & -14.93 & 20.53 & -15.29 & 20.93 & -15.66 \\
\hline .74 & -14.57 & 20.14 & $-14 \cdot 94$ & $20 \cdot 54$ & $-15 \cdot 30$ & 20.94 & -15.67 \\
\hline & -14.58 & 20.15 & $-14 \cdot 94$ & 20.55 & $-15 \cdot 31$ & 20.95 & -15.68 \\
\hline .76 & -14.59 & 20.16 & -14.95 & 20.56 & -15.32 & 20.96 & $-15 \cdot 68$ \\
\hline .77 & -14.60 & 20.17 & -14.96 & 20.57 & -15.33 & 20.97 & -15.69 \\
\hline .78 & -14.61 & 20.18 & -14.97 & 20.58 & $-15 \cdot 34$ & 20.98 & -15.70 \\
\hline 79 & $-14 \cdot 62$ & $20 \cdot 19$ & $-14 \cdot 98$ & 20.59 & $-15 \cdot 35$ & 20.99 & -15.71 \\
\hline 80 & -14.63 & 20.20 & -14.99 & 20.60 & -15.35 & 21.00 & -15.72 \\
\hline & -14.64 & 20.21 & -15.00 & 20.61 & -15.36 & 21.01 & -15.73 \\
\hline 82 & -14.64 & 20.22 & -15.01 & 20.62 & -15.37 & 21.02 & -15.74 \\
\hline & -14.65 & 20.23 & -15.02 & 20.63 & -15.38 & 21.03 & -15.75 \\
\hline 34 & -14.66 & 20.24 & -15.03 & 20.64 & -15.39 & 21.04 & $-15 \cdot 76$ \\
\hline 85 & -14.67 & 20.25 & -15.04 & 20.65 & -15.40 & 21.05 & -15.77 \\
\hline & -14.68 & 20.26 & -15.04 & 20.66 & -15.41 & 21.06 & -15.78 \\
\hline 87 & -14.69 & 20.27 & $-15.05^{\circ}$ & 20.67 & $-15 \cdot 42$ & 21.07 & -15.79 \\
\hline 88 & -14.70 & 20.28 & -15.06 & 20.68 & -15.43 & 21.08 & -15.79 \\
\hline 89 & -14.71 & 20.29 & -15.07 & 20.69 & $-15 \cdot 44$ & 21.09 & $-15 \cdot 80$ \\
\hline 90 & -14.72 & $20 \cdot 30$ & -15.08 & 20.70 & -15.45 & $21 \cdot 10$ & -15.81 \\
\hline 91 & -14.73 & $20 \cdot 31$ & -15.09 & 20.71 & -15.46 & 21.11 & -15.82 \\
\hline 92 & -14.73 & 20.32 & -15.10 & 20.72 & -15.46 & 21.12 & -15.83 \\
\hline & -14.74 & 20.33 & -15.11 & 20.73 & -15.47 & 21.13 & $-15 \cdot 84$ \\
\hline 94 & -14.75 & 20.34 & -15.12 & 20.74 & -15.48 & $21 \cdot 14$ & -15.85 \\
\hline & -14.76 & 20.35 & -15.13 & 20.75 & -15.49 & 21.15 & -15.86 \\
\hline 96 & -14.77 & 20.36 & $-15 \cdot 14$ & 20.76 & $-15 \cdot 50$ & 21.16 & -15.87 \\
\hline & -14.78 & $20 \cdot 37$ & $-15 \cdot 14$ & 20.77 & -15.51 & 21.17 & $-15 \cdot 88$ \\
\hline & -14.79 & $20 \cdot 38$ & -15.15 & 20.78 & -15.52 & 21.18 & -15.89 \\
\hline & -14.80 & 20.39 & $-15 \cdot 16$ & 20.79 & -15.53 & 21.19 & -15.90 \\
\hline & -14.81 & 20.40 & -15.17 & 20.80 & -15.54 & 21.20 & -15.91 \\
\hline
\end{tabular}




\begin{tabular}{|c|c|c|c|c|c|c|c|}
\hline$A_{0}-A_{r}$ & A & $A_{0}-A_{r}$ & A & $A_{0}-A_{r}$ & A & $A_{0}-A_{r}$ & A \\
\hline 21.21 & -15.91 & 21.61 & $-16 \cdot 28$ & 22.01 & -16.65 & 22.41 & -17.02 \\
\hline $21 \cdot 22$ & -15.92 & 21.62 & -16.29 & 22.02 & -16.66 & 22.42 & -17.03 \\
\hline 21.23 & $-15 \cdot 93$ & 21.63 & $-16 \cdot 30$ & 22.03 & -16.67 & 22.43 & -17.04 \\
\hline 21.24 & -15.94 & 21.64 & $-16 \cdot 31$ & 22.04 & $-16 \cdot 68$ & 22.44 & -17.05 \\
\hline 21.25 & -15.95 & 21.65 & $-16 \cdot 32$ & 22.05 & -16.69 & $22 \cdot 45$ & -17.06 \\
\hline 21.26 & -15.96 & 21.66 & $-16 \cdot 33$ & 22.06 & -16.70 & 22.46 & -17.07 \\
\hline 21.27 & -15.97 & 21.67 & $-16 \cdot 34$ & 22.07 & -16.71 & 22.47 & -17.08 \\
\hline $21 \cdot 28$ & $-15 \cdot 98$ & $21 \cdot 68$ & $-16 \cdot 35$ & 22.08 & -16.72 & 22.48 & -17.09 \\
\hline 21.29 & -15.99 & 21.69 & $-16 \cdot 36$ & 22.09 & -16.73 & 22.49 & $-17 \cdot 10$ \\
\hline 21.30 & -16.00 & 21.70 & $-16 \cdot 37$ & 22.10 & -16.74 & 22.50 & -17.11 \\
\hline $21 \cdot 31$ & -16.01 & 21.71 & $-16 \cdot 38$ & $22 \cdot 11$ & -16.75 & $22 \cdot 51$ & $-17 \cdot 12$ \\
\hline 21.32 & -16.02 & 21.72 & $-16 \cdot 38$ & 22.12 & -16.75 & 22.52 & -17.13 \\
\hline 21.33 & -16.02 & 21.73 & -16.39 & 22.13 & $-16 \cdot 76$ & 22.53 & -17.14 \\
\hline 21.34 & -16.03 & 21.74 & $-16 \cdot 40$ & 22.14 & -16.77 & 22.54 & -17.15 \\
\hline 21.35 & -16.04 & 21.75 & $-16 \cdot 41$ & 22.15 & $-16 \cdot 78$ & 22.55 & $-17 \cdot 15$ \\
\hline 21.36 & -16.05 & 21.76 & $-16 \cdot 42$ & 22.16 & $-16 \cdot 79$ & 22.56 & $-17 \cdot 16$ \\
\hline 21.37 & -16.06 & 21.77 & $-16 \cdot 43$ & 22.17 & $-16 \cdot 80$ & 22.57 & -17.17 \\
\hline $21 \cdot 38$ & -16.07 & 21.78 & -16.44 & 22.18 & $-16 \cdot 81$ & 22.58 & -17.18 \\
\hline 21.39 & -16.08 & 21.79 & $-16 \cdot 45$ & 22.19 & $-16 \cdot 82$ & 22.59 & $-17 \cdot 19$ \\
\hline $21 \cdot 40$ & -16.09 & 21.80 & $-16 \cdot 46$ & 22.20 & -16.83 & 22.60 & $-17 \cdot 20$ \\
\hline 21.41 & $-16 \cdot 10$ & 21.81 & $-16 \cdot 47$ & 22.21 & $-16 \cdot 84$ & 22.61 & $-17 \cdot 21$ \\
\hline $21 \cdot 42$ & -16.11 & 21.82 & $-16 \cdot 48$ & 22.22 & -16.85 & 22.62 & $-17 \cdot 22$ \\
\hline $21 \cdot 43$ & -16.12 & 21.83 & $-16 \cdot 49$ & 22.23 & $-16 \cdot 86$ & 22.63 & -17.23 \\
\hline $21 \cdot 44$ & -16.13 & 21.84 & $-16 \cdot 50$ & 22.24 & -16.87 & $22 \cdot 64$ & $-17 \cdot 24$ \\
\hline $21 \cdot 45$ & $-16 \cdot 14$ & 21.85 & -16.50 & 22.25 & $-16 \cdot 88$ & 22.65 & -17.25 \\
\hline $21 \cdot 46$ & -16.14 & 21.86 & $-16 \cdot 51$ & 22.26 & $-16 \cdot 88$ & 22.66 & $-17 \cdot 26$ \\
\hline 21.47 & $-16 \cdot 15$ & 21.87 & -16.52 & 22.27 & $-16 \cdot 89$ & 22.67 & $-17 \cdot 27$ \\
\hline 21.48 & $-16 \cdot 16$ & 21.88 & $-16 \cdot 53$ & 22.28 & $-16 \cdot 90$ & 22.68 & $-17 \cdot 28$ \\
\hline $21 \cdot 49$ & $-16 \cdot 17$ & 21.89 & $-16 \cdot 54$ & 22.29 & -16.91 & 22.69 & $-17 \cdot 28$ \\
\hline $21 \cdot 50$ & $-16 \cdot 18$ & 21.90 & -16.55 & $22 \cdot 30$ & $-16 \cdot 92$ & 22.70 & $-17 \cdot 29$ \\
\hline 21.51 & $-16 \cdot 19$ & 21.91 & $-16 \cdot 56$ & 22.31 & $-16 \cdot 93$ & 22.71 & $-17 \cdot 30$ \\
\hline 21.52 & $-16 \cdot 20$ & 21.92 & $-16 \cdot 57$ & $22 \cdot 32$ & $-16 \cdot 94$ & 22.72 & $-17 \cdot 31$ \\
\hline 21.53 & -16.21 & 21.93 & $-16 \cdot 58$ & 22.33 & -16.95 & 22.73 & $-17 \cdot 32$ \\
\hline 21.54 & $-16 \cdot 22$ & 21.94 & $-16 \cdot 59$ & 22.34 & $-16 \cdot 96$ & 22.74 & $-17 \cdot 33$ \\
\hline 21.55 & $-16 \cdot 23$ & 21.95 & -16.60 & 22.35 & -16.97 & 22.75 & $-17 \cdot 34$ \\
\hline 21.56 & $-16 \cdot 24$ & 21.96 & -16.61 & 22.36 & $-16 \cdot 98$ & 22.76 & -17.35 \\
\hline 21.57 & -16.25 & 21.97 & $-16 \cdot 62$ & $22 \cdot 37$ & -16.99 & 22.77 & $-17 \cdot 36$ \\
\hline 21.58 & $-16 \cdot 26$ & 21.98 & $-16 \cdot 63$ & $22 \cdot 38$ & -17.00 & 22.78 & -17.37 \\
\hline 21.59 & $-16 \cdot 26$ & 21.99 & $-16 \cdot 63$ & 22.39 & -17.01 & 22.79 & $-17 \cdot 38$ \\
\hline 21.60 & $-16 \cdot 27$ & 22.00 & $-10 \cdot 64$ & 22.40 & -17.01 & 22.80 & -17.39 \\
\hline
\end{tabular}


$A_{0}-A_{r}$

A

$A_{0}-A_{r}$

A

$A_{0}-A_{r}$

A

$A_{0}-A_{x}$

A
$22.81-17.40$

$22.82-17.41$

$22.83-17.42$

$22.84-17.42$

$22.85-17.43$

$22.86-17.44$

$22.87-17.45$

$22.88-17.46$

$22.89-17.47$

22. $90-17.48$

$22.91-17.49$

$22.92-17.50$

$22.93-17.51$

$22.94-17.52$

$22.95-17.53$

$22.46-17.54$

$22.97-17.55$

$22.98-17.56$

$22.99-17.56$

$23.00-17.57$

$23.01-17.58$

$23.02-17.59$

$23.03-17.60$

$23.04-17.61$

$23.05-17.62$

$23.06-17.63$

$23.07-17.64$

$23.08-17.65$

$23.09-17.66$

$23.10-17.67$

$23.11-17.68$

$23.12-17.69$

$23.13-17.70$

$23.14-17.70$

$23.15-17.71$

$23.16-17.72$

$23.17-17.73$

$23.18-17.74$

$23.19-17.75$

$23.20-17.76$
$23.21-17.77$

$23.22-17.78$

$23.23-17.79$

$23.24-17.80$

$23.25-17.81$

$23.26-17.32$

$23.27-17.83$

$23.28-17.84$

$23.29-17.84$

$23.30-17.85$

$23.31-17.86$

$23.32-17.87$

$23.33-17.88$

$23.34-17.89$

$23.35-17.90$

$23.36-17.91$

$23.37-17.92$

$23.38-17.93$

$23.39-17.94$

$23.40-17.95$

$23.41-17.96$

$23.42-17.97$

$23.43-17.98$

$23.44-17.99$

$23.45-17.99$

$23.46-18.00$

$23.47-18.01$

$23.48-18.02$

$23.49-18.03$

$23.50-18.04$

$23.51-18.05$

$23.52-18.06$

$23.53-18.07$

$23.54-18.08$

$23.55-18.09$

$23.56-18.10$

$23.57-18.11$

$23.58-18.12$

$23.59-18.13$

$23.60-18.14$
$23.61-18.14$

$23.62-18.15$

$23.63-18.16$

$23.64-18.17$

$23.65-18.18$

$23.66-18.19$

$23.67-18.20$

$23.68-18.21$

$23.69-18.22$

$23.70-18.23$

$23.71-18.24$

$23.72-18.25$

$23.73-18.26$

$23.74-18.27$

$23.75-18.28$

$23.76-18.29$

$23.77-18.30$

$23.78-18.30$

$23.79-18.31$

$23.80-18.32$

$23.81-18.33$

$23.82-18.34$

$23.83-18.35$

$23.84-18.36$

$23.85-18.37$

$23.86-18.38$

$23.87-18.39$

$23.88-18.40$

$23.89-18.41$

$23.90-18.42$

$23.91-18.43$

$23.92-18.44$

$23.93-18.45$

$23.94-18.45$

$23.95-18.46$

$23.96-18.47$

$23.97-18.48$

$23.98-18.49$

$23.99-18.50$

$24.00-18.51$
$24.01-18.52$

$24.02-18.53$

$24.03-18.54$

$24.04-18.55$

$24.05-18.56$

$24.06-18.57$

$24.07-18.58$

$24.08-18.59$

$24.09-18.60$

$24.10-18.61$

$24.11-18.61$

$24.12-18.62$

$24.13-18.63$

$24.14-18.64$

$24.15-18.65$

$24.16-18.66$

$24.17-18.67$

$24.18-18.68$

$24.19-18.69$

$24.20-18.70$

$24.21-18.71$

$24.22-18.72$

$24.23-18.73$

$24.24-18.74$

$24.25-18.75$

$24.26-18.76$

$24.27-18.77$

$24.28-18.77$

$24.29-18.78$

$24.30-18.79$

$24.31-18.80$

$24.32-18.81$

$24.33-18.82$

$24.34-18.83$

$24.35-18.84$

$24.36-18.85$

$24.37-18.86$

$24.38-18.87$

$24.39-18.88$

$24.40-18.89$ 

$A_{0}-A_{r}$
A
$A_{0}-A_{r}$
A
$A_{0}-A_{r}$
A
$A_{0}{ }^{-A_{r}}$
A

\begin{tabular}{|c|c|c|c|c|c|c|c|}
\hline & & & & & & & \\
\hline 4.41 & 18.90 & 24.81 & 19.28 & 25.21 & -19.65 & 25.61 & -20 \\
\hline $4 \cdot 42$ & -18.91 & 24.82 & -19.28 & 25.22 & -19.66 & 25.62 & -20 \\
\hline 4.43 & -18.92 & 24.83 & -19.29 & 25.23 & -19.67 & 25.63 & -20 \\
\hline 4.44 & -18.93 & 24.84 & $-19 \cdot 30$ & 25.24 & -19.68 & $25 \cdot 64$ & \\
\hline 4.45 & $-18 \cdot 94$ & 24.85 & $-19 \cdot 31$ & 25.25 & -19.69 & 25.65 & -20 \\
\hline 24.46 & $-18 \cdot 94$ & 24.86 & -19.32 & 25.26 & -19.70 & 25.66 & -20 \\
\hline 24.47 & -18.95 & 24.87 & -19.33 & 25.27 & -19.71 & 25.67 & -20 \\
\hline 24.48 & -18.96 & 24.88 & -19.34 & 25.28 & -19.72 & 25.68 & -20 \\
\hline 24.49 & -18.97 & 24.89 & -19.35 & 25.29 & -19.73 & 25.69 & -20 \\
\hline 24.50 & -18.98 & 24.90 & $-19 \cdot 36$ & 25.30 & -19.74 & 25.70 & -20 \\
\hline 24.51 & $-18 \cdot 99$ & 24.91 & -19.37 & 25.31 & -19.75 & 25.71 & -2 \\
\hline 4.52 & -19.00 & 24.92 & $-19 \cdot 38$ & 25.32 & -19.76 & 25.72 & -2 \\
\hline 24.53 & -19.01 & 24.93 & $-19 \cdot 39$ & 25.33 & -19.77 & 25.73 & \\
\hline 24.54 & -19.02 & $24 \cdot 94$ & -19.40 & $25 \cdot 34$ & -19.78 & 25.74 & -20 \\
\hline 24.55 & -19.03 & 24.95 & -19.41 & 25.35 & -19.79 & 25.75 & - \\
\hline 4.56 & -19.04 & 24.96 & $-19 \cdot 42$ & 25.36 & $-19 \cdot 80$ & 25.76 & -20 \\
\hline 4.57 & -19.05 & 24.97 & $-19 \cdot 43$ & 25.37 & -19.81 & 25.77 & -20 \\
\hline 24.58 & -19.06 & 24.98 & $-19 \cdot 44$ & 25.38 & $-19 \cdot 82$ & 25.78 & \\
\hline 24.59 & -19.07 & 24.99 & $-19 \cdot 45$ & 25.39 & -19.82 & 25.79 & -20 \\
\hline 24.60 & -19.08 & 25.00 & $-19 \cdot 45$ & 25.40 & $-19 \cdot 83$ & 25.80 & -20 \\
\hline 4.61 & -19.09 & 25.01 & $-19 \cdot 46$ & 25.41 & $-19 \cdot 84$ & 25.81 & -20 \\
\hline 24.62 & -19.10 & 25.02 & 47 & 42 & -19.85 & 25.82 & -2 \\
\hline 24.63 & -19.11 & 25.03 & $-19 \cdot 48$ & 25.43 & $-19 \cdot 86$ & 25.83 & -20 \\
\hline 24.64 & -19.11 & 25.04 & -19.49 & 25.44 & -19.87 & 25.84 & -20 \\
\hline 24.65 & -19.12 & 25.05 & $-19 \cdot 50$ & 25.45 & -19.88 & 25.85 & -20 \\
\hline 24.66 & -19.13 & 25.06 & -19.51 & 46 & -19.89 & 25.86 & \\
\hline 24.67 & -19.14 & 25.07 & -19.52 & 25.47 & $-19 \cdot 90$ & 25.87 & -20 \\
\hline 24.68 & -19.15 & 25.08 & -19.53 & 25.48 & -19.91 & 25.88 & -20 \\
\hline 24.69 & -19.16 & 25.09 & -19.54 & 25.49 & -19.92 & 25.89 & -20 \\
\hline 24.70 & $-19 \cdot 17$ & $25 \cdot 10$ & -19.55 & 25.50 & -19.93 & 25.90 & -2 \\
\hline 24.71 & $-19 \cdot 18$ & 25.11 & -19.56 & 25.51 & -19.94 & 25.91 & -20 \\
\hline 24.72 & $-19 \cdot 19$ & 25.12 & -19.57 & 25.52 & -19.95 & 25.92 & -20 \\
\hline 24.73 & -19.20 & 25.13 & $-19 \cdot 58$ & 25.53 & $-19 \cdot 96$ & 25.93 & -20 \\
\hline 24.74 & $-19 \cdot 21$ & $25 \cdot 14$ & $-19 \cdot 59$ & 25.54 & -19.97 & 25.94 & -20 \\
\hline 24.75 & -19.22 & 25.15 & $-19 \cdot 60$ & 25.55 & -19.98 & 25.95 & -20 \\
\hline 24.76 & 9.23 & 25.16 & -19.61 & 25.56 & -19.99 & 25.96 & -20 \\
\hline 24.77 & 9.24 & 25.17 & -19.62 & 25.57 & $-20 \cdot 00$ & 25.97 & -20 \\
\hline 24.78 & 19.25 & $25 \cdot 18$ & $-19 \cdot 63$ & 25.58 & -20.01 & 25.98 & -20 \\
\hline 4.79 & .9 .26 & 25.19 & -19.63 & 25.59 & -20.01 & 25.99 & -20 \\
\hline 24.80 & 9.27 & 25.20 & -19.64 & 25.60 & -20.02 & 26.00 & -20 \\
\hline
\end{tabular}


$A_{0}-A_{r}$

A

$A_{0}-A_{r}$

A

$A_{0}-A_{r}$

A

$\mathrm{A}_{0}{ }^{-A_{r}}$

A
$26.01-20.41$

$26.02-20.42$

$26.03-20.43$

$26.04-20.44$

$26.05-20.45$

$26.06-20.46$

$26.07-20.47$

$26.08-20.48$

$26.09-20.49$

$26.10-20.50$

$26.11-20.51$

$26.12-20.52$

$26.13-20.53$

$26.14-20.54$

$26.15-20.55$

$26.16-20.56$

$26.17-20.57$

$26.18-20.58$

$26.19-20.59$

$26.20-20.60$

$26.21-20.60$

$26.22-20.61$

$26.23-20.62$

$26.24-20.63$

$26.25-20.64$

$26.26-20.65$

$26.27-20.66$

$26.28-20.67$

$26.29-20.68$

$26.30-20.69$

$26.31-20.70$

$26.32-20.71$

$26.33-20.72$

$26.34-20.73$

$26.35-20.74$

$26.36-20.75$

$26.37-20.76$

$26.38-20.77$

$26.39-20.78$

$26.40-20.79$
$26.41-20.80$

$26.42-20.80$

$26.43-20.81$

$26.44-20.82$

$26.45-20.83$

$26.46-20.84$

$26.47-20.85$

$26.48-20.86$

$26.49-20.87$

$26.50-20.88$

$26.51-20.89$

$26.52-20.90$

$26.53-20.91$

$26.54-20.92$

$26.55-20.93$

$26.56-20.94$

$26.57-20.95$

$26.58-20.96$

$26.59-20.97$

$26.60-20.98$

$26.61-20.99$

$26.62-21.00$

$26.63-21.01$

$26.64-21.01$

$26.65-21.02$

$26.66-21.03$

$26.67-21.04$

$26.68-21.05$

$26.69-21.06$

$26.70-21.07$

$26.71-21.08$

$26.72-21.09$

$26.73-21.10$

$26.74-21.11$

$26.75-21.12$

$26.76-21.13$

$26.77-21.14$

$26.78-21.15$

$26.79-21.16$

$26.80-21 \cdot 17$
$26.81-21.18$

$26.82-21.19$

$26.83-21.20$

$26.84-21.21$

$26.85-21.22$

$26.86-21.23$

$26.87-21.23$

$26.88-21.24$

$26.89-21.25$

$26.90-21.26$

$26.91-21.27$

$26.92-21.28$

$26.93-21.29$

$26.94-21.30$

$26.95-21.31$

$26.96-21.32$

$26.97-21.33$

$26.98-21.34$

$26.99-21.35$

$27.00-21.36$

$27.01-21.37$

$27.02-21.38$

$27.03-21.39$

$27.04-21.40$

$27.05-21.41$

$27.06-21.42$

$27.07-21.43$

$27.08-21.44$

$27.09-21.45$

$27.10-21.46$

$27.11-21.46$

$27.12-21.47$

$27.13-21.48$

$27.14-21.49$

$27.15-21.50$

$27.16-21.51$

$27.17-21.52$

$27.18-21.53$

$27.19-21.54$

$27.20-21.55$
$27.21-21.56$

$27.22-21.57$

$27.23-21.58$

$27.24-21.59$

$27.25-21.60$

$27.26-21.61$

$27.27-21.62$

$27.28-21.63$

$27.29-21.64$

$27.30-21.65$

$27.31-21.66$

$27.32-21.67$

$27.33-21.68$

$27.34-21.69$

$27.35-21.69$

$27.36-21.70$

$27.37-21.71$

$27.38-21.72$

$27.39-21.73$

$27.40-21.74$

$27.41-21.75$

$27.42-21.76$

$27.43-21.77$

$27.44-21.78$

$27.45-21.79$

$27.46-21.80$

$27.47-21.81$

$27.48-21.82$

$27.49-21.83$

$27.50-21.84$

$27.51-21.85$

$27.52-21.86$

$27.53-21.87$

$27.54-21.88$

$27.55-21.89$

$27.56-21.90$

$27.57-21.91$

$27.58-21.92$

$27.59-21.92$

$27.60-21.93$ 


$A_{0}-A_{r} \quad A \quad A_{0}-A_{r} \quad A \quad A_{0}-A_{r} \quad A \quad A_{0}-A_{r} \quad A$

\begin{tabular}{|c|c|c|c|c|c|c|c|}
\hline 27.61 & $-21 \cdot 94$ & 28.01 & $-22 \cdot 33$ & 28.41 & -22.71 & 28.81 & $-23 \cdot 10$ \\
\hline 27.62 & -21.95 & 28.02 & $-22 \cdot 34$ & 28.42 & -22.72 & 28.82 & -23.11 \\
\hline 7.63 & -21.96 & 28.03 & $-22 \cdot 35$ & 28.43 & -22.73 & 28.83 & -23.1 \\
\hline 27.64 & -21.97 & 28.04 & $-22 \cdot 36$ & 28.44 & -22.74 & 28.84 & -23.1 \\
\hline 7.65 & $-21 \cdot 98$ & 28.05 & $-22 \cdot 37$ & 28.45 & -22.75 & 28.85 & -23.1 \\
\hline & -21.99 & 28.06 & $-22 \cdot 38$ & 28.46 & -22.76 & 28.86 & - \\
\hline .7 .67 & -22.00 & 28.07 & $-22 \cdot 39$ & 28.47 & -22.77 & 28.87 & -23.1 \\
\hline 27.68 & -22.01 & 28.08 & $-22 \cdot 40$ & 28.48 & -22.78 & 28.88 & -23.1 \\
\hline 7.69 & -22.02 & 28.09 & $-22 \cdot 41$ & 28.49 & -22.79 & 28.89 & -23.1 \\
\hline .7 .70 & -22.03 & 28.10 & $-22 \cdot 41$ & 28.50 & $-22 \cdot 80$ & 28.90 & \\
\hline$: 7.71$ & -22.04 & 28.11 & $-22 \cdot 42$ & 28.51 & -22.81 & 28.91 & -23.2 \\
\hline 27.72 & -22.05 & 28.12 & $-22 \cdot 43$ & 28.52 & -22.82 & 28.92 & -23 \\
\hline 27.73 & -22.06 & 28.13 & -22.44 & 28.53 & -22.83 & 28.93 & -23.2 \\
\hline 27.74 & -22.07 & 28.14 & -22.45 & 28.54 & -22.84 & 28.94 & -23.2 \\
\hline 27.75 & -22.08 & 28.15 & $-22 \cdot 46$ & 28.55 & -22.85 & 28.95 & -23.2 \\
\hline 27.76 & -22.09 & 28.16 & $-22 \cdot 47$ & 28.56 & -22.86 & 28.96 & \\
\hline 27.77 & $-22 \cdot 10$ & 28.17 & $-22 \cdot 48$ & 28.57 & -22.87 & 28.97 & -23.2 \\
\hline 27.78 & -22.11 & 28.18 & -22.49 & 28.58 & $-22 \cdot 38$ & 28.98 & -23.2 \\
\hline 27.79 & -22.12 & 28.19 & $-22 \cdot 50$ & 23.59 & -22.89 & 28.99 & -23.2 \\
\hline 27.80 & -22.13 & 28.20 & $-22 \cdot 51$ & 28.60 & $-22 \cdot 90$ & 29.00 & \\
\hline 7.81 & -22.14 & 28.21 & $-22 \cdot 52$ & 28.61 & $-22 \cdot 91$ & 29.01 & -23.2 \\
\hline 7.82 & $-22 \cdot 15$ & 28.22 & -22.53 & 28.62 & $-22 \cdot 92$ & 29.02 & $-23 \cdot 3$ \\
\hline 27.83 & -22.16 & 28.23 & -22.54 & 28.63 & -22.93 & 29.03 & -23.3 \\
\hline 84 & $-22 \cdot 16$ & 28.24 & $-22 \cdot 55$ & 28.64 & 94 & 29.04 & \\
\hline 7.85 & -22.17 & 28.25 & $-22 \cdot 56$ & 28.65 & $-22 \cdot 94$ & 29.05 & -23.3 \\
\hline 7.86 & -22.18 & 28.26 & -22.57 & 28.66 & $-22 \cdot 95$ & 29.06 & $-23 \cdot 3$ \\
\hline 27.87 & $-22 \cdot 19$ & 28.27 & $-\overline{2} 2.58$ & 28.67 & $-22 \cdot 96$ & 29.07 & -2 \\
\hline 27.88 & $-22 \cdot 20$ & 28.28 & $-22 \cdot 59$ & 28.68 & -22.97 & 29.08 & -23 \\
\hline 7.89 & -22.21 & 28.29 & -22.60 & 28.69 & $-22 \cdot 98$ & 29.09 & $-23 \cdot 3$ \\
\hline 7.90 & -22.22 & 28.30 & $-22 \cdot 61$ & 28.70 & -22.99 & 29.10 & -23.3 \\
\hline 27.91 & -22.23 & 28.31 & $-22 \cdot 62$ & 28.71 & -23.00 & 29.11 & \\
\hline 7.92 & -22.24 & 28.32 & -22.63 & 28.72 & -23.01 & 29.12 & -23.4 \\
\hline 7.93 & $-22 \cdot 25$ & 28.33 & -22.64 & 28.73 & -23.02 & 29.13 & -23.4 \\
\hline 27.94 & -22.26 & 28.34 & -22.65 & 28.74 & -23.03 & 29.14 & -2 \\
\hline 27.95 & -22.27 & 28.35 & $-22 \cdot 66$ & 28.75 & -23.04 & 29.15 & \\
\hline 96 & $-22 \cdot 28$ & 28.36 & -22.67 & 28.76 & -23.05 & 29.16 & -23 \\
\hline .7 .97 & -22.29 & 28.37 & -22.67 & 28.77 & -23.06 & 29.17 & -2 \\
\hline 7.98 & $-22 \cdot 30$ & 28.38 & $-22 \cdot 68$ & 28.78 & -23.07 & 29.18 & -23 \\
\hline 99 & 22.31 & 28.39 & -22.69 & 28.79 & -23.08 & 29.19 & \\
\hline 8.00 & $-22 \cdot 32$ & 28.40 & -22.70 & 28.80 & -23.09 & 29.20 & -2 \\
\hline
\end{tabular}


$A_{0}-A_{r}$

A

$A_{0}-A_{r}$

A

$29.21-23.49$

$29.22-23.50$

$29.23-23.50$

$29.24-23.51$

$29.25-23.52$

$29.26-23.53$

$29.27-23.54$

$29.28-23.55$

$29.29-23.56$

$29.30-23.57$

$29.31-23.58$

$29.32-23.59$

$29.33-23.60$

$29.34-23.61$

$29.35-23.62$

$29.36-23.63$

$29.37-23.64$

$29.38-23.65$

$29.39-23.66$

$29.40-23.67$

$29.41-23.68$

$29.42-23.69$

$29.43-23.70$

$29.44-23.71$

$29.45-23.72$

$29.46-23.73$

$29.47-23.74$

$29.48-23.75$

$29.49-23.76$

$29.50-23.77$

$29.51-23.78$

$29.52-23.79$

$29.53-23.79$

$29.54-23.80$

$29.55-23.81$

$29.56-23.82$

$29.57-23.83$

$29.58-23.84$

$29.59-23.85$

$29.60-23.86$

$29.61-23.87$
$29.62-23.88$
$29.63-23.89$
$29.64-23.90$
$29.65-23.91$
$29.66-23.92$
$29.67-23.93$
$29.68-23.94$
$29.69-23.95$
$29.70-23.96$
$29.71-23.97$
$29.72-23.98$
$29.73-23.99$
$29.74-24.00$
$29.75-24.01$
$29.76-24.02$
$29.77-24.03$
$29.78-24.04$
$29.79-24.05$
$29.80-24.06$
$29.81-24.07$
$29.82-24.08$
$29.83-24.09$
$29.84-24.10$
$29.85-24.10$
$29.86-24.11$
$29.87-24.12$
$29.88-24.13$
$29.89-24.14$
$29.90-24.15$
$29.91-24.16$
$29.92-24.17$
$29.93-24.18$
$29.94-24.19$
$29.95-24.20$
$29.96-24.21$
$29.97-24.22$
$29.98-24.23$
$29.99-24.24$
$30.00-24.25$

$A_{0}-A_{r}$

A

$A_{0}-A_{r}$

A

$\begin{array}{llll}30.01 & -24.26 & 30.41 & -24.65 \\ 30.02 & -24.27 & 30.42 & -24.66 \\ 30.03 & -24.28 & 30.43 & -24.67 \\ 30.04 & -24.29 & 30.44 & -24.68 \\ 30.05 & -24.30 & 30.45 & -24.69 \\ 30.06 & -24.31 & 30.46 & -24.70 \\ 30.07 & -24.32 & 30.47 & -24.71 \\ 30.08 & -24.33 & 30.48 & -24.72 \\ 30.09 & -24.34 & 30.49 & -24.73 \\ 30.10 & -24.35 & 30.50 & -24.74 \\ 30.11 & -24.36 & 30.51 & -24.74 \\ 30.12 & -24.37 & 30.52 & -24.75 \\ 30.13 & -24.38 & 30.53 & -24.76 \\ 30.14 & -24.39 & 30.54 & -24.77 \\ 30.15 & -24.40 & 30.55 & -24.78 \\ 30.16 & -24.41 & 30.56 & -24.79 \\ 30.17 & -24.41 & 30.57 & -24.80 \\ 30.18 & -24.42 & 30.58 & -24.81 \\ 30.19 & -24.43 & 30.59 & -24.82 \\ 30.20 & -24.44 & 30.60 & -24.83 \\ 30.21 & -24.45 & 30.61 & -24.84 \\ 30.22 & -24.46 & 30.62 & -24.85 \\ 30.23 & -24.47 & 30.63 & -24.86 \\ 30.24 & -24.48 & 30.64 & -24.87 \\ 30.25 & -24.49 & 30.65 & -24.88 \\ 30.26 & -24.50 & 30.66 & -24.89 \\ 30.27 & -24.51 & 30.67 & -24.90 \\ 30.28 & -24.52 & 30.68 & -24.91 \\ 30.29 & -24.53 & 30.69 & -24.92 \\ 30.30 & -24.54 & 30.70 & -24.93 \\ 30.31 & -24.55 & 30.71 & -24.94 \\ 30.32 & -24.56 & 30.72 & -24.95 \\ 30.33 & -24.57 & 30.73 & -24.96 \\ 30.34 & -24.58 & 30.74 & -24.97 \\ 30.35 & -24.59 & 30.75 & -24.98 \\ 30.36 & -24.60 & 30.76 & -24.99 \\ 30.37 & -24.61 & 30.77 & -25.00 \\ 30.38 & -24.62 & 30.78 & -25.01 \\ 30.39 & -24.63 & 30.79 & -25.02 \\ 30.40 & -24.64 & 30.80 & -25.03\end{array}$

$30.01-24.26$

$30.41-24.65$

$30.42-24.66$

$30.43-24.67$

$30.44-24.68$

$30.45-24.69$

$30.46-24.70$

$30.47-24.71$

$30.48-24.72$

$30.49-24.73$

$30.50-24.74$

$30.51-24.74$

$30.53-24.76$

$30.54-24.77$

$30.55-24.78$

$30.56-24.79$

$30.57-24.80$

$30.58-24.81$

$30.59-24.82$

$30.60-24.83$

$30.61-24.84$

$30.62-24.85$

$30.63-24.86$

$30.65-24.88$

$30.66-24.89$

$30.67-24.90$

$30.68-24.91$

$30.69-24.92$

$30.71-24.94$

$30.72-24.95$

$30.73-24.96$

$30.74-24.97$

$30.75-24.98$

$30.76-24.99$

$30.78-25.01$

$30.80-25.03$ 


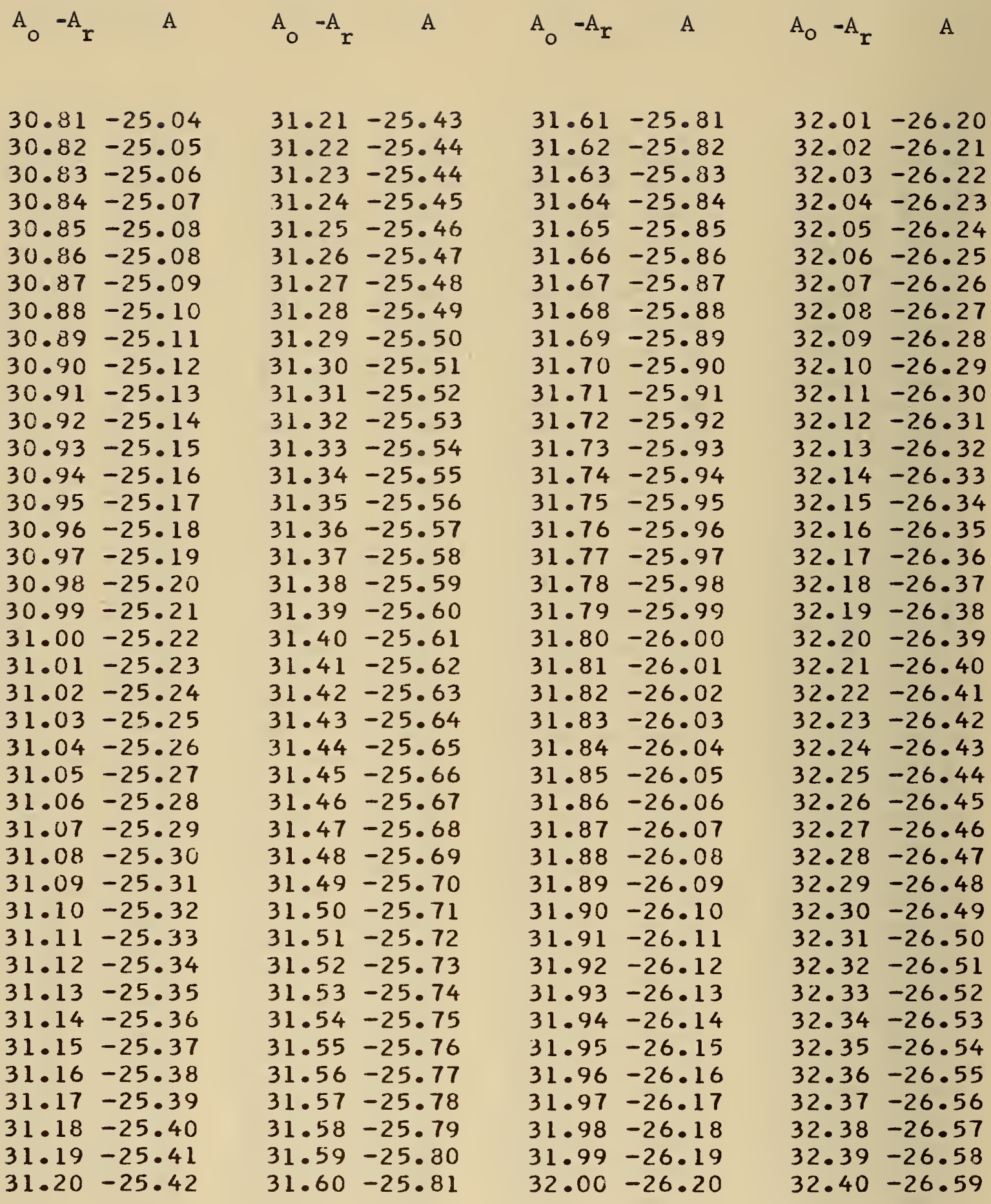





\section{THE NATIONAL BUREAU OF STANDARDS}

The scope of activities of the National Bureau of Standards at its major laboratories in Washington, D.C., and Boulder, Colorado, is suggested in the following listing of the divisions and sections engaged in technical work. In general, each section carries out specialized research, development, and engineering in the field indicated by its title. A brief description of the activities, and of the resultant publications, appears on the inside of the front cover.

\section{WASHINGTON, D. C.}

Electricity. Resistance and Reactance. Electrochemistry. Electrical lnstruments. Magnetic Measurements Dielectrics. High Voltage.

Metrology. Photometry and Colorimetry. Refractometry. Photographic Research. Length. Engineering Metrology. Mass and Scale. Volumetry and Densimetry.

Heat. Temperature Physics. Heat Measurements. Cryogenic Physics. Equation of State. Statistical Physics. Radiation Physics. X-ray. Radioactivity. Radiation Theory. High Energy Radiation. Radiological Equipment. Nucleonic Instrumentation. Neutron Physics.

Analytical and Inorganic Chemistry. Pure Substances. Spectrochemistry. Solution Chemistry. Standard Reference Materials. Applied Analytical Research. Crystal Chemistry.

Mechanics. Sound. Pressure and Vacuum. Fluid Mechanics. Engineering Mechanics. Rheology. Combustion Controls.

Polymers. Macromolecules: Synthesis and Structure. Polymer Chemistry. Polymer Physics. Polymer Characterization. Polymer Evaluation and Testing. Applied Polymer Standards and Research. Dental Research.

Metallurgy, Engineering Metallurgy. Microscopy and Diffraction. Metal Reactions. Metal Physics. Electrolysis and Metal Deposition.

Inorganic Solids. Engineering Ceramics. Glass. Solid State Chemistry. Crystal Growth. Physical Properties. Crystallography.

Building Research. Structural Engineering. Fire Research. Mechanical Systems. Organic Building Materials. Codes and Safety Standards. Heat Transfer. Inorganic Building Materials. Metallic Building Materials.

Applied Mathematics. Numerical Analysis. Computation. Statistical Engineering. Mathematical Physics. Operations Research.

Data Processing Systems. Components and Techniques. Computer Technology. Measurements Automation. Engineering Applications. Systems Analysis.

Atomic Physics. Spectroscopy. Infrared Spectroscopy. Far Ulitraviolet Physics. Solid State Physics. Electron Physics. Atomic Physics. Plasma Spectroscopy.

Instrumentation. Engineering Electronics. Electron Devices. Electronic lnstrumentation. Mechanical lnstruments. Basic lnstrumentation.

Physical Chemistry. Thermochemistry. Surface Chemistry. Organic Chemistry. Molecular Spectroscopy. Elementary Processes. Mass Spectrometry. Photochemistry and Radiation Chemistry.

office of Weights and Measures.

BOULDER, COLO.

Cryogenic Engineering Laboratory. Cryogenic Equipment. Cryogenic Processes. Properties of Materials. Cryogenic Technical Services.

\section{CENTRAL, RADIO PROPAGATION LABORATORY}

lonosphere Research and Propagation. Low Frequency and Very Low Frequency Research. Ionosphere Research. Prediction Services. Sun-Earth Relationships. Field Engineering. Radio Warning Services. Vertical Soundings Research.

Radio Propagation Engineering. Data Reduction Instrumentation. Radio Noise. Tropospheric Measurements. Tropo \$pheric Analysis. Propagation-Terrain Effects. Radio-Meteorology. Lower Atmosphere Physics.

Radio Systems. Applied Flectromagnetic Theory. High Frequency and Very High Frequency Research. Frequency Utilization. Modulation Research. Antenna Research. Radiodetermination.

Upper Atmosphere and Space Physics. Upper Atmosphere and Plasma Physics. High Latitude lonosphere Physics. lonosphere and Exosphere Scatter. Airglow and Aurora. Ionospheric Radio Astronomy.

\section{RADIO STANDARDS LABORATORY}

Radio Physics. Radio Broadcast Service. Radio and Microwave Materials. Atomic Frequency and Time-Interval Standards. Radio Plasma. Millimeter-Wave Research.

Circuit Standards. High Frequency Electrical Standards. High Frequency Calibration Services. High Frequency Impedance Standards. Microwave Calibration Services. Microwave Circuit Standards. Low Frequency Calibration Services. 


\section{NBS}

